\title{
ON THE VARIANCE OF THE ERROR TERM IN THE HYPERBOLIC CIRCLE PROBLEM
}

\author{
GIACOMO CHERUBINI AND MORTEN S. RISAGER
}

\begin{abstract}
Let $e(s)$ be the error term of the hyperbolic circle problem, and denote by $e_{\alpha}(s)$ the fractional integral to order $\alpha$ of $e(s)$. We prove that for any small $\alpha>0$ the asymptotic variance of $e_{\alpha}(s)$ is finite, and given by an explicit expression. Moreover, we prove that $e_{\alpha}(s)$ has a limiting distribution.
\end{abstract}

\section{INTRODUCTION}

Let $\mathbb{H}$ be the hyperbolic plane, and denote by $d(z, w)$ be the hyperbolic distance between $z, w \in \mathbb{H}$. For $\Gamma$ a cofinite Fuchsian group and $z, w \in \mathbb{H}$, consider the function

$$
N(s, z, w):=\sharp\{\gamma \in \Gamma \mid d(z, \gamma w) \leq s\},
$$

which counts the number of translates $\gamma w$ of $w, \gamma \in \Gamma$ with hyperbolic distance from the point $z$ not exceeding $s$. The hyperbolic lattice point problem asks for the behaviour of $N(s, z, w)$ for big values of $s$. It is known that

$$
N(s, z, w) \sim \frac{\operatorname{vol}\left(B_{z}(s)\right)}{\operatorname{vol}(\Gamma \backslash \mathbb{H})}
$$

as $s \rightarrow \infty$. Here $B_{z}(s)$ denotes the hyperbolic ball with center $z$ and radius $s$. This can be proved in several ways, see e.g. [8, Section 1.3].

For our purposes it is convenient to appeal to the spectral theory of the LaplaceBeltrami operator

$$
\Delta=-y^{2}\left(\frac{\partial^{2}}{\partial x^{2}}+\frac{\partial^{2}}{\partial y^{2}}\right)
$$

acting on a dense subset of $L^{2}(\Gamma \backslash \mathbb{H})$. The operator $\Delta$ has a discrete spectrum

$$
0=\lambda_{0}<\lambda_{1} \leq \lambda_{2} \leq \lambda_{3} \leq \cdots
$$

which is either finite or satisfies $\lambda_{n} \rightarrow \infty$, and a continuous spectrum which covers $[1 / 4, \infty)$ with multiplicity equal to the number of cusps of $\Gamma$. The eigenvalues $\lambda_{j} \in(0,1 / 4)$ are called small eigenvalues. Writing $\lambda_{j}=1 / 4+t_{j}^{2}$ with $\Im\left(t_{j}\right) \geq 0$ they correspond to $t_{j}$ in the complex segment $t_{j} \in(0, i / 2)$. One defines the following

Date: October 7, 2018.

2000 Mathematics Subject Classification. Primary 11P21, 11F72; Secondary .

Key words and phrases. Hyperbolic lattice points, Selberg's pre-trace formula, Fractional integration.

Both author were supported by a Sapere Aude grant from The Danish Council for Independent Research (Grant-id:0602-02161B). 
main term:

$$
\begin{gathered}
\left.M(s, z, w):=\frac{\pi e^{s}}{\operatorname{vol}(\Gamma \backslash \mathbb{H})}+\sqrt{\pi} \sum_{t_{j} \in\left(0, \frac{i}{2}\right)} \frac{\Gamma\left(\left|t_{j}\right|\right)}{\Gamma\left(3 / 2+\left|t_{j}\right|\right)} e^{s\left(1 / 2+\left|t_{j}\right|\right)} \phi_{j}(z) \overline{\phi_{j}(w}\right) \\
+4(s+2(\log 2-1)) e^{s / 2} \sum_{t_{j}=0} \phi_{j}(z) \overline{\phi_{j}(w)} \\
\left.+e^{s / 2} \sum_{\mathfrak{a}} E_{\mathfrak{a}}(z, 1 / 2) \overline{E_{\mathfrak{a}}(w, 1 / 2}\right)
\end{gathered}
$$

where $\phi_{j}$ is the eigenfunction associated to $\lambda_{j}$, and $E_{\mathfrak{a}}(z, r)$ is the Eisenstein series associated to the cusp $\mathfrak{a}$. For the full modular group this expression simplifies to only the first term, but for general groups the small eigenvalues give rise to secondary terms in the expansion of the counting function $N(s, z, w)$. It is an unpublished result of Selberg (for a proof see e.g.[10, Thm. 12.1]) that

$$
N(s, z, w)-M(s, z, w) \ll e^{\frac{2 s}{3}},
$$

and it is conjectured that the true size of the difference should be not bigger than $e^{s\left(\frac{1}{2}+\varepsilon\right)}$ for any $\varepsilon>0$. Define

$$
e_{\Gamma}(s, z, w)=\frac{N(s, z, w)-M(s, z, w)}{e^{s / 2}}
$$

to be the normalized remainder in the hyperbolic problem.

Phillips and Rudnick have shown ([13, Theorem 1.1]) that

$$
\lim _{T \rightarrow \infty} \frac{1}{T} \int_{0}^{T} e_{\Gamma}(s, z, z) d s=0 .
$$

We refer to the quantity on the left as the first (asymptotic) moment of $e_{\Gamma}(s, z, z)$.

It is an open problem whether the (asymptotic) variance of $e_{\Gamma}(s, z, w)$ exists and, if so, if it is finite. More precisely we are interested in knowing if

$$
\operatorname{Var}\left(e_{\Gamma}\right)=\lim _{T \rightarrow \infty} \frac{1}{T} \int_{T}^{2 T}\left|e_{\Gamma}(s, z, w)\right|^{2} d s
$$

exists and is finite. Phillips and Rudnick remarked [13, Section 3.8] that they cannot show that the variance is finite but they prove non-zero lower bounds.

In order to simplify notation, from now on we will write $e(s)$ in place of $e_{\Gamma}(s, z, w)$, assuming that the group $\Gamma$ and the points $z, w \in \mathbb{H}$ are fixed once and for all.

A first result on the size of the variance is due to Chamizo see [4, Corollary 2.1.1] who proves, using his large sieve in Riemann surfaces [3]

$$
\frac{1}{T} \int_{T}^{2 T}|e(s)|^{2} d s \ll T^{2}
$$

(one gets from his statement to (1.5) by changing variable $X=2 \cosh (s)$ ). This doesn't prove finiteness of the variance of $e(s)$ but does shows that the integral in (1.5) grows at most polynomially in $T$. This is an improvement on what one gets by simply plugging Selberg's pointwise bound, and it is consistent with the conjecture $e(s) \ll e^{\varepsilon s}$. We remark that (1.5) can be improved to a bound $\ll T$ by using classical methods due to Cramér [6, 7]. For details see [5].

REMARK 1.1. Cramér studied the analogous Euclidian problem [7], and in this case he was able to prove that the variance is finite and find an explicit expression for it. Like us, he also used a spectral expansion (coming in his case from Poisson summation), but contrary to our case the "eigenvalues" are explicitly known and the decay of the spectral coefficients is favorable. One difficulty in proving finiteness of the variance in our problem (using a spectral approach) relates to the 
following feature of the problem: the spectral coefficients do not decay sufficiently fast compared to the number of eigenvalues. The way we get around this problem is to slightly improve the decay of the coefficients using fractional integration. The formalism that we adopt follows the lines of [15].

Definition 1.2. Let $\varphi \in L^{p}([0, A])$ be a $p$-summable function on $[0, A]$ for $p \geq 1$, and let $\alpha>0$ be a positive real number. The fractional integral of order $\alpha$ of $\varphi$ is defined for $x \in[0, A]$ as the function

$$
I_{\alpha} \varphi(x)=\frac{1}{\Gamma(\alpha)} \int_{0}^{x} \frac{\varphi(t)}{(x-t)^{1-\alpha}} d t .
$$

The function $I_{\alpha} \varphi(x)$ will also be denoted by $\varphi_{\alpha}(x)$.

It is straightforward from the definition that the fractional integral of order $\alpha=1$ coincides with the regular integral. It is interesting to consider integrals of small order $0<\alpha<1$ of a given function $\varphi$, because we have

$$
\begin{gathered}
\lim _{\alpha \rightarrow 0^{+}} \varphi_{\alpha}(x)=\varphi(x) \quad \text { for a.e. } x \in[0, A] . \\
\lim _{\alpha \rightarrow 0^{+}}\left\|\varphi_{\alpha}-\varphi\right\|_{p}=0 .
\end{gathered}
$$

The first condition is easy to check by integration by parts when $\varphi$ is regular. If we integrate the function $\varphi$ to a very small order, we expect thus the resulting function $\varphi_{\alpha}$ to be close to the original function. In addition to this, fractional integration enhances the properties of $\varphi$; Indeed, if $0<\alpha<1$ and $\varphi \in L^{p}$ with $1<p<1 / \alpha$, then $\varphi_{\alpha} \in L^{q}$, for $q=p /(1-\alpha p)>p$, and therefore $\varphi_{\alpha}$ has better summability properties. Moreover, if $\varphi \in L^{\infty}$, then $\varphi_{\alpha}$ is Hölder of exponent $\alpha$, and in general, if $\varphi$ is Hölder of exponent $0 \leq \rho \leq 1$, then for $0<\alpha<1$ the function $\varphi_{\alpha}$ is Hölder of exponent $\rho+\alpha^{1}$. Hence $\varphi_{\alpha}$ has better regularity properties than $\varphi$. For a reference on these and other results about fractional integration, see [15].

Definition 1.3. Let $0<\alpha<1$. We define, for $s>0$, the $\alpha$-integrated normalized remainder term in the hyperbolic lattice point counting problem as

$$
e_{\alpha}(s, z, w):=I_{\alpha} e_{\Gamma}(s, z, w) .
$$

where the integration is with respect to the first $s$ variable.

The function $e_{\alpha}(s, z, w)$ is well-defined since for every $A>0$ we have $e(s) \in$ $L^{1}([0, A])$. When the group $\Gamma$ and the points $z, w \in \mathbb{H}$ are fixed, we will simply write $e_{\alpha}(s)$.

We first prove a pointwise bound and an average result for $e_{\alpha}(s)$ that are analogous to the results for $e(s)$ :

Theorem 1.4. Let $\Gamma$ be a cofinite group, $z, w \in \mathbb{H}$, and $0<\alpha<1$. Then

$$
e_{\alpha}(s) \ll \begin{cases}e^{s(1-2 \alpha) /(6-4 \alpha)} & 0<\alpha<1 / 2, \\ s & \alpha=1 / 2, \\ 1 & 1 / 2<\alpha<1 .\end{cases}
$$

The implied constant depends on $z, w$, and the group $\Gamma$.

REMARK 1.5. When $\alpha=0$ this is Selberg's bound (1.3) (recall the normalization in (1.4)). When $\alpha>0$ the exponent gets smaller approaching 0 as $\alpha$ increases to $1 / 2$. For the threshold $\alpha=1 / 2$ a polynomial factor appears, while for $\alpha>1 / 2$ the function $e_{\alpha}(s)$ becomes bounded.

\footnotetext{
1 The case $\rho+\alpha=1$ is special, as in this situation $\varphi_{\alpha}$ is in a slightly bigger space than $H^{1}$ (see [15, Ch. 1, §3.3, Cor 1]).
} 
Theorem 1.6. Let $\Gamma$ be a cofinite group, $z, w \in \mathbb{H}$, and $0<\alpha<1$. Then

$$
\lim _{T \rightarrow \infty} \frac{1}{T} \int_{T}^{2 T} e_{\alpha}(s) d s=0
$$

REMARK 1.7. When $\alpha=0$ this corresponds to [13, Theorem 1.1]. The case $\alpha=1$ is delicate: if the group is cofinite but not cocompact we cannot show that the limit stays bounded, while if the group is cocompact then it is possible to show that the limit exists and is finite.

To be able to prove finite variance for $e_{\alpha}(s)$ we need to make assumptions on the Eisenstein series. More precisely we need to assume, in the case where $\Gamma$ is cofinite but not cocompact, that for $v=z$ and $v=w$ we have

(1.6) $\int_{1}^{\infty} \frac{\left|E_{\mathfrak{a}}(v, 1 / 2+i t)\right|^{2 p}}{t^{(3 / 2+\alpha) p}} d t<\infty, \quad$ for some $1<p<\min \left(2, \alpha^{-1}\right)$, and all $\mathfrak{a}$.

Theorem 1.8. Let $0<\alpha<1$ and assume (1.6). Then we have

$$
\lim _{T \rightarrow \infty} \frac{1}{T} \int_{T}^{2 T}\left|e_{\alpha}(s)\right|^{2} d s=2 \pi \sum_{\substack{t_{j}>0 \\ \text { distinct }}} \frac{\left|\Gamma\left(i t_{j}\right)\right|^{2}}{\left|t_{j}^{\alpha} \Gamma\left(3 / 2+i t_{j}\right)\right|^{2}}\left|\sum_{t_{j^{\prime}}=t_{j}} \phi_{j^{\prime}}(z) \overline{\phi_{j^{\prime}}(w)}\right|^{2}
$$

and the sum on the right is convergent.

REMARK 1.9. Condition (1.6) holds true for congruence groups: it is implied by the following stronger condition

$$
\left|E_{\mathfrak{a}}(z, 1 / 2+i t)\right| \ll_{z}|t|^{1 / 2+\varepsilon}, \quad t \gg 1
$$

which holds for congruence groups. For a proof see [16, Lemma 2.1] or combine [2, Eq. (2.4), ftnote 2.] with a Maass-Selberg type argument as in the proof of [14, Lemma 6.1].

For cocompact groups (1.6) is vacuous, so Theorem 1.8 holds unconditional in this case.

Condition (1.6) holds also if $\alpha>1 / 2$ and the Eisenstein series satisfy that they are bounded polynomially as $t \rightarrow \infty$. We note also, by using Theorem 1.4 , that when $\alpha>1 / 2$ the asymptotic variance is bounded.

REMARK 1.10. It is a straightforward exercise to show that if $f \in L_{l o c}^{1}([0, \infty))$ then $T^{-1} \int_{T}^{2 T} f(s) d s \rightarrow A$ as $T \rightarrow \infty$ if and only if $T^{-1} \int_{0}^{T} f(s) d s \rightarrow A$ as $T \rightarrow \infty$. It follows that theorems 1.6 and 1.8 are true also if we replace the integral from $T$ to $2 T$ by the integral from 0 to $T$. For various technical reasons it is convenient to consider the integral from $T$ to $2 T$.

REMARK 1.11. If we take $\alpha=0$ we cannot prove that the infinite series appearing in Theorem 1.8 is convergent. However, for groups like $\mathrm{SL}_{2}(\mathbb{Z})$ this follows from standard (but probably very hard) conjectures (see Section 8 ). For groups $\Gamma$ where

$$
V=2 \pi \sum_{\substack{t_{j}>0 \\ \text { distinct }}} \frac{\left|\Gamma\left(i t_{j}\right)\right|^{2}}{\left|\Gamma\left(3 / 2+i t_{j}\right)\right|^{2}}\left|\sum_{t_{j^{\prime}}=t_{j}} \phi_{j^{\prime}}(z) \overline{\phi_{j^{\prime}}(w)}\right|^{2}<\infty
$$

and where the Eisenstein contribution is "small" it is tempting to speculate that V should be the variance of $e_{\Gamma}(s)$ i.e. that

$$
\operatorname{Var}\left(e_{\Gamma}\right)=\lim _{\alpha \rightarrow 0^{+}} \operatorname{Var}\left(e_{\alpha}\right)=V .
$$

In fact, by comparison of (1.7) with the explicit expression of the variance of error terms in other problems (see $[6,7,1]$ ), the quantity $V$ seems the appropriate candidate for being the variance of $e_{\Gamma}$. 
Finally, we conclude with a distributional result on $e_{\alpha}(s)$ which we prove as a by-product of bounds which emerge in the proof Theorem 1.8. Given a function $g: \mathbb{R}_{\geq 0} \rightarrow \mathbb{R}$ we say that $g$ admits a limiting distribution if there exists a probability measure $\mu$ on $\mathbb{R}$ such that

$$
\lim _{T \rightarrow \infty} \frac{1}{T} \int_{0}^{T} f(g(s)) d s=\int_{\mathbb{R}} f d \mu
$$

holds for every bounded continuous function $f: \mathbb{R} \rightarrow \mathbb{R}$.

Theorem 1.12. Let $0<\alpha<1$ and let $\Gamma$ be as in Theorem 1.8. Then the function $e_{\alpha}(s)$ admits a limiting distribution $\mu_{\alpha}$. For $\alpha>1 / 2, \mu_{\alpha}$ is compactly supported.

In view of Remark 1.9, the theorem applies to congruence groups and cocompact groups.

REMARK 1.13. The technique of regularizing functions that do not have sufficiently good properties is standard in analytic number theory. This can often be done for instance by convolution with some smooth functions $f_{\varepsilon}$ that approximate a delta function as $\varepsilon$ tends to zero, and it is in particular this type of smoothing that is used in [13] when proving lower bounds on $e(s)$. Using fractional integration corresponds to pushing the standard method to its limit. Indeed, the pre-trace formula for the integrated function $e_{\alpha}(s)$ for $\alpha \leq 1 / 2$ is not absolutely convergent, which is a characteristic of $e(s)$ but not of the smooth approximation. The small improvements given by the $\alpha$ integration allows to prove the above theorems.

\section{Preliminaries}

We recall here some basic facts on automorphic functions. Let $z, w \in \mathbb{H}$, and consider the standard point-pair invariant

$$
u(z, w)=\frac{|z-w|^{2}}{4 \Im(z) \Im(w)} .
$$

We have

$$
2 u(z, w)+1=\cosh d(z, w)
$$

Let $\Gamma \leq \operatorname{PSL}(2, \mathbb{R})$ be a cofinite Fuchsian group. For $k: \mathbb{R} \rightarrow \mathbb{R}$ rapidly decreasing the function

$$
K(z, w)=\sum_{\gamma \in \Gamma} k(u(z, \gamma w))
$$

is an automorphic kernel for the group $\Gamma$. If we define $h(t)$ to be the SelbergHarish-Chandra transform of $k(u)$, which is defined as an integral transform of $k$ in three steps as follows

$$
q(v)=\int_{v}^{+\infty} \frac{k(u)}{(u-v)^{1 / 2}} d u, \quad g(r)=2 q\left(\sinh ^{2} \frac{r}{2}\right), \quad h(t)=\int_{-\infty}^{+\infty} e^{i r t} g(r) d r
$$

then we have the following spectral expansion of $K(z, w)$, usually referred to as the pre-trace formula (see [10, Theorem 7.4]):

Proposition 2.1. Let $(k, h)$ be a pair such that $h(t)$ is even, holomorphic on a strip $|\Im(t)| \leq 1 / 2+\varepsilon$, and with $h(t) \ll(1+|t|)^{-2-\varepsilon}$ in the strip. We have the following expansion for $K(z, w)$ :

$$
K(z, w)=\sum_{t_{j}} h\left(t_{j}\right) \phi_{j}(z) \overline{\phi_{j}(w)}+\frac{1}{4 \pi} \sum_{\mathfrak{a}} \int_{\mathbb{R}} h(t) E_{\mathfrak{a}}(z, 1 / 2+i t) \overline{E_{\mathfrak{a}}(w, 1 / 2+i t)} d t
$$

and the right hand side converges absolutely and uniformly on compact sets. 
The absolute convergence is a consequence of the local Weyl's law (See e.g. [13, Lemma 2.3])

$$
\sum_{\left|t_{j}\right|<T}\left|\phi_{j}(z)\right|^{2}+\frac{1}{4 \pi} \sum_{\mathfrak{a}} \int_{-T}^{T}\left|E_{\mathfrak{a}}(z, 1 / 2+i t)\right|^{2} d t \sim c T^{2}
$$

as $T \rightarrow \infty$, for a positive constant $c>0$, together with the assumption on the decay of $h$.

Using the pre-trace formula it is possible to give upper bounds on eigenfunctions averaged over short intervals. More precisely one can show (see [10, Eq. (13.8)])

$$
\sum_{T \leq t_{j} \leq T+1}\left|\phi_{j}(z)\right|^{2} \ll T \text {. }
$$

In our proofs we also need to consider

$$
b_{j}=\sum_{t_{j^{\prime}}=t_{j}} \phi_{j^{\prime}}(z) \overline{\phi_{j^{\prime}}(w)} \ll t_{j}
$$

where the bound follows immediately from (2.3). From (2.2) we find immediately that

$$
\sum_{T \leq t_{j} \leq 2 T}^{\prime}\left|b_{j}\right| \ll T^{2}
$$

Here and in the rest of the paper a prime on a sum indexed over $t_{j}$ means that in this sum the $t_{j}$ are listed without multiplicity, i.e. $t_{j} \neq t_{\ell}$ for $j \neq \ell$.

Consider the counting function defined in (1.1). This can be written as an automorphic kernel as

$$
N(s, z, w)=\sum_{\gamma \in \Gamma} k_{s}(u(z, \gamma w))
$$

where $k_{s}(u)=\mathbf{1}_{[0,(\cosh s-1) / 2]}$ is the indicator function of the set $[0,(\cosh s-1) / 2]$. This agrees with (1.1) by virtue of (2.1). In particular

$$
k_{s}(u(z, w))= \begin{cases}1 & \text { if } d(z, w) \leq s \\ 0 & \text { if } d(z, w)>s .\end{cases}
$$

To study the error term $e(s, z, w)$ we want to use the pre-trace formula. However the Selberg-Harish-Chandra transform of $k_{s}$ only decays as fast as $O\left((1+|t|)^{-3 / 2}\right)$ (see [13, Lemma 2.5 and 2.6]) and the pre-trace formula is therefore not absolutely convergent. The standard way to go around this is by regularizing the function $k_{s}$ sufficiently to ensure that the associated Selberg-Harish-Chandra transform has better decay properties.

Since our purpose is to study the normalized remainder, we consider instead of $k_{s}(u)$ the function $k_{s}(u) e^{-s / 2}$. This gives rise to the function $N(s, z, w) e^{-s / 2}$, and subtracting from it the normalized main term $M(s, z, w) e^{-s / 2}$ we obtain $e_{\Gamma}(s, z, w)$. The fractional integral of order $\alpha$ of $e_{\Gamma}(s, z, w)$ is defined by $e_{\alpha}(s)=I_{\alpha} e_{\Gamma}(s, z, w)$. By linearity of the fractional integral we see that

$$
e_{\alpha}(s)=I_{\alpha} e_{\Gamma}(s, z, w)=I_{\alpha}\left(\frac{N(s, z, w)}{e^{s / 2}}\right)-I_{\alpha}\left(\frac{M(s, z, w)}{e^{s / 2}}\right) .
$$

It is easy to compute directly what the second term is. We have indeed for $\beta>0$

$$
I_{\alpha}\left(e^{\beta s}\right)=\frac{e^{\beta s}}{\beta^{\alpha}}+O\left(\frac{1}{\beta \Gamma(\alpha) s^{1-\alpha}}\right)
$$

and

$$
I_{\alpha}(s)=\frac{s^{\alpha+1}}{\Gamma(\alpha+2)}, \quad I_{\alpha}(1)=\frac{s^{\alpha}}{\Gamma(\alpha+1)} .
$$


The implied constant in the first expression is absolute. It is now natural to define the $\alpha$-integrated normalized main term to be

$$
\begin{aligned}
M_{\alpha}(s):= & \left.\frac{\pi e^{s / 2}}{2^{-\alpha} \operatorname{vol}(\Gamma \backslash \mathbb{H})}+\sqrt{\pi} \sum_{t_{j} \in\left(0, \frac{i}{2}\right)} \frac{\Gamma\left(\left|t_{j}\right|\right)}{\left|t_{j}\right|^{\alpha} \Gamma\left(3 / 2+\left|t_{j}\right|\right)} e^{s\left|t_{j}\right|} \phi_{j}(z) \overline{\phi_{j}(w}\right) \\
& +4\left(\frac{s^{\alpha+1}}{\Gamma(\alpha+2)}+\frac{2(\log 2-1) s^{\alpha}}{\Gamma(\alpha+1)}\right) \sum_{t_{j}=0} \phi_{j}(z) \overline{\phi_{j}(w)} \\
& \left.+\frac{s^{\alpha}}{\Gamma(\alpha+1)} \sum_{\mathfrak{a}} E_{\mathfrak{a}}(z, 1 / 2) \overline{E_{\mathfrak{a}}(w, 1 / 2}\right) .
\end{aligned}
$$

We then have

$$
M_{\alpha}(s)=I_{\alpha}\left(\frac{M(s, z, w)}{e^{s / 2}}\right)+O\left(\frac{1}{\Gamma(\alpha) s^{1-\alpha}}\right)
$$

where the implied constant depends on $z, w$, and the group $\Gamma$. We conclude that the $\alpha$-integrated normalized remainder is expressed as

$$
e_{\alpha}(s)=N_{\alpha}(s)-M_{\alpha}(s)+O\left(\frac{1}{\Gamma(\alpha) s^{1-\alpha}}\right) .
$$

The function $N_{\alpha}(s)=I_{\alpha}\left(N(s, z, w) e^{-s / 2}\right)$ can be expressed as an automorphic function associated to the kernel $k_{\alpha}(u)=I_{\alpha}\left(k_{s}(u) e^{-s / 2}\right)$ in the following way:

$$
N_{\alpha}(s)=I_{\alpha}\left(\frac{N(s, z, w)}{e^{s / 2}}\right)=I_{\alpha}\left(\sum_{\gamma \in \Gamma} \frac{k_{s}(u(z, w))}{e^{s / 2}}\right)=\sum_{\gamma \in \Gamma} k_{\alpha}(u(z, w)) .
$$

Here we have used that the sum is finite, so we can interchange the order of integration and summation. In order to apply the pre-trace formula we need to understand the properties of the Selberg-Harish-Chandra transform $h_{\alpha}^{\prime}(t)$ associated to $k_{\alpha}(u)$. We have the following expression (see [10, (1.62')]) for the Selberg-Harish-Chandra transform of a generic test function $k(u)$ :

$$
h(t)=4 \pi \int_{0}^{+\infty} F_{1 / 2+i t}(u) k(u) d u,
$$

where $F_{\nu}(u)$ is the hypergeometric function. It follows, using that $k_{s}$ is compactly supported in $u$, that

$$
\begin{aligned}
h_{\alpha}^{\prime}(t) & =4 \pi \int_{0}^{+\infty} F_{1 / 2+i t}(u) k_{\alpha}(u) d u \\
& =4 \pi \int_{0}^{+\infty} F_{1 / 2+i t}(u) I_{\alpha}\left(k_{s}(u) e^{-s / 2}\right) d u=I_{\alpha}\left(h_{s}(t) e^{-s / 2}\right)
\end{aligned}
$$

where we have used that $I_{\alpha} k_{s}(u)$ is an integral in the variable $s$, and the double integral is absolutely convergent so that we can interchange the order of integration. The function $h_{\alpha}^{\prime}(t)$ is the spectral function associated to the remainder $e_{\alpha}(s)$. We study in detail this function in section 3.

\section{Analysis of the Selberg-Harish-Chandra transform}

We prove some estimates on the function $I_{\alpha}\left(h_{s}(t) e^{-s / 2}\right)$ that will be useful to prove pointwise and average results for the remainder function $e_{\alpha}(s)$. 
3.1. Integral representation. The Selberg-Harish-Chandra transform $h_{s}^{\prime}(t)$ of the kernel $k_{s}^{\prime}(u)=\mathbf{1}_{[0,(\cosh s-1) / 2]}(u) e^{-s / 2}$ is given for $s \geq 0$ by (see [13, (2.10)] and $[4,(2.6)])$

$$
h_{s}^{\prime}(t)=\frac{2^{3 / 2}}{e^{s / 2}} \int_{-s}^{s}(\cosh s-\cosh r)^{1 / 2} e^{i r t} d r .
$$

It is an important but non-obvious feature of $h_{s}^{\prime}(t)$ that it decays as fast as $|t|^{-3 / 2}$ when $t \rightarrow \infty$. A flexible method to show such decay consists in shifting the contour of integration from the interval $[-s, s]$ to a pair of vertical half-lines in the complex plane, with base points $\pm s$. This is done in [13, Lemma 2.5], and this method can be used also to analyse the fractional integral of $h_{s}^{\prime}$. Since the function $h_{s}^{\prime}(t)$ is even, we will from now on only consider $t>0$, using the reflection formula $h_{s}^{\prime}(t)=h_{s}^{\prime}(-t)$ to get negative values of $t$. We have (see [13, p. 89])

$$
\begin{gathered}
h_{s}^{\prime}(t)=2 \Re\left(J_{s}(t)\right), \\
J_{s}(t)=-2 i \int_{0}^{\infty}\left(1-e^{i v}\right)^{1 / 2}\left(1-e^{-2 s} e^{-i v}\right)^{1 / 2} e^{-t v} d v e^{i t s} .
\end{gathered}
$$

It is convenient also to set $J_{s}(t)=0$ for $s<0$. For technical reasons that will be clear later, it is convenient to consider a small shift of the function $h_{s}^{\prime}(t)$. For $0 \leq \delta<1$ and $s>2$ consider the function $h_{s \pm \delta}^{\prime}(t)$. We consider the fractional integral of order $\alpha$ of $h_{s \pm \delta}^{\prime}(t)$, which we will denote by $h_{\alpha, s \pm \delta}^{\prime}(t)$, i.e

$$
\begin{aligned}
h_{\alpha, s \pm \delta}^{\prime}(t) & =\frac{1}{\Gamma(\alpha)} \int_{0}^{s} \frac{2 \Re\left(J_{x \pm \delta}(t)\right)}{(s-x)^{1-\alpha}} d x \\
(3.1) & =2 \Re\left(\frac{-2 i}{\Gamma(\alpha)} \int_{0}^{\infty}\left(1-e^{i v}\right)^{1 / 2} e^{-t v} \int_{s_{0}}^{s} \frac{\left(1-e^{-2(x \pm \delta)-i v}\right)^{1 / 2} e^{i t(x \pm \delta)}}{(s-x)^{1-\alpha}} d x d v\right) .
\end{aligned}
$$

Here $s_{0}$ denotes the quantity $s_{0}=\max \{0, \mp \delta\}$. We will consider the innermost integral and move the contour of integration to two vertical half-lines in the upper half-plane of $\mathbb{C}$ with base points $s_{0}, s$. In order to move the contour we define for $\varepsilon>0$ the set

$$
\Omega_{\varepsilon}=\left\{z \in \mathbb{C}: z=x+i y, s_{0}<x<s, y>0,|z-s|>\varepsilon\right\} .
$$

The integrand

$$
f(z)=\frac{\left(1-e^{-2(z \pm \delta)-i v}\right)^{1 / 2} e^{i t(z \pm \delta)}}{(s-z)^{1-\alpha}}
$$

is holomorphic on $\Omega_{\varepsilon}$ and continuous on its boundary, so we can apply Cauchy's theorem and get

$$
\int_{s_{0}}^{s-\varepsilon} f(z) d z=\int_{\ell_{1}} f(z) d z-\int_{\ell_{2, \varepsilon}} f(z) d z-\int_{\gamma_{\varepsilon}} f(z) d z
$$

where $\ell_{1}=\left\{z=s_{0}+i y, y \geq 0\right\}, \ell_{2, \varepsilon}=\left\{z \in \partial \Omega_{\varepsilon}, \Re(z)=s,|z-s| \geq \varepsilon\right\}$ and $\gamma_{\varepsilon}=\left\{z \in \partial \Omega_{\varepsilon}:|z-s|=\varepsilon\right\}$. Since we can bound

$$
\left|\int_{\gamma_{\varepsilon}} f(z) d z\right| \leq \frac{\pi \varepsilon^{\alpha}}{\sqrt{2}}
$$

we see, taking the limit as $\varepsilon \rightarrow 0$ in (3.2), that the integral over $\left[s_{0}, s\right]$ equals the integral over $\ell_{1}$ minus the integral over $\ell_{2}=\{s+i y, y \geq 0\}$. This gives

$$
\begin{aligned}
& \Gamma(\alpha) h_{\alpha, s \pm \delta}^{\prime}(t) \\
&=2 \Re\left(2 \int_{0}^{\infty}\left(1-e^{i v}\right)^{1 / 2} e^{-t v} \int_{0}^{\infty} \frac{\left(1-e^{-2\left(s_{0} \pm \delta+i \lambda / t\right)-i v}\right)^{1 / 2} e^{-\lambda}}{\left(\left(s-s_{0}\right) t-i \lambda\right)^{1-\alpha}} \frac{d \lambda d v}{t^{\alpha}} e^{i t\left(s_{0} \pm \delta\right)}\right) \\
&-2 \Re\left(2 \int_{0}^{\infty}\left(1-e^{i v}\right)^{1 / 2} e^{-t v} \int_{0}^{\infty} \frac{\left(1-e^{-2(s \pm \delta+i \lambda / t)-i v}\right)^{1 / 2} e^{-\lambda}}{(-i \lambda)^{1-\alpha}} \frac{d \lambda d v}{t^{\alpha}} e^{i t(s \pm \delta)}\right) .
\end{aligned}
$$


In the rest of the section we will use this integral representation of $h_{\alpha, s \pm \delta}^{\prime}(t)$ to obtain pointwise bounds for $h_{\alpha, s \pm \delta}^{\prime}(t)$, bounds for the average $\frac{1}{T} \int_{T}^{2 T} h_{\alpha, s \pm \delta}^{\prime}(t) d s$, and for products $\frac{1}{T} \int_{T}^{2 T} h_{\alpha, s \pm \delta}^{\prime}\left(t_{1}\right) \overline{h_{\alpha, s \pm \delta}^{\prime}\left(t_{2}\right)} d s$. These will be used in section 5 to get pointwise estimates on $e_{\alpha}(s)$, and in sections 6 and 7 to get estimates for the first and second moment of $e_{\alpha}(s)$.

In several of the proofs we will tacitly use the following elementary inequalities to interpolate between different bounds:

$$
\begin{aligned}
\min \left(a^{-1}, b^{-1}\right) & \leq 2 /(a+b) \leq 2 \min \left(a^{-1}, b^{-1}\right) \\
\min (c, d) & \leq c^{\sigma} d^{1-\sigma}
\end{aligned}
$$

valid for all $a, b, c, d>0$ and $0 \leq \sigma \leq 1$.

3.2. Pointwise bounds. We now state and prove two lemmas in which we estimate pointwise the function $h_{\alpha, s \pm \delta}^{\prime}(t)$. One is uniform in $t$ but worse in $s$, while the second is sharper for $|t| \gg 1$ but it has a singularity when $t \rightarrow 0$.

Lemma 3.1. Let $0<\alpha<1$ and let $t \in \mathbb{R}$. For $0 \leq \delta<1$ and $s>2$ we have

$$
h_{\alpha, s \pm \delta}^{\prime}(t) \ll s^{\alpha+1}
$$

where the implied constant is absolute.

Proof. Since the function $h_{s}^{\prime}(t)$ satisfies the bound $\left|h_{s}^{\prime}(t)\right| \leq\left|h_{s}^{\prime}(0)\right| \ll s$ for every $t \in \mathbb{R}$ (see [13, Lemma 2.2]) and in view of the fact that fractional integration preserves inequalities, we get for every $t \in \mathbb{R}$

$$
h_{\alpha, s \pm \delta}^{\prime}(t)=I_{\alpha}\left(h_{s \pm \delta}^{\prime}(t)\right) \ll I_{\alpha}(s)=\frac{s^{\alpha+1}}{\Gamma(\alpha+2)}
$$

where the last equality follows from (2.7).

Lemma 3.2. Let $0<\alpha<1$ and $t \in \mathbb{R}, t \neq 0$. Then for $0 \leq \delta<1$ and $s>2$

$$
h_{\alpha, s \pm \delta}^{\prime}(t)=2 \sqrt{\pi} \Re\left(\frac{\Gamma(i t) e^{i t(s \pm \delta)}}{(i t)^{\alpha} \Gamma(3 / 2+i t)}\right)+\ell(s, \delta, t)
$$

where

$$
\ell(s, \delta, t)=O\left(\frac{1}{|t|^{1+\alpha}(1+\sqrt{|t|})}\left(e^{-2 s}+\frac{1}{\left(1+|s t|^{1-\alpha} \Gamma(\alpha)\right)}\right)\right)
$$

and the implied constant is absolute.

Proof. We have from Section 3.1 that $h_{\alpha, s \pm \delta}^{\prime}(t)=2 \Re\left(L_{1}\right)+2 \Re\left(L_{2}\right)+2 \Re\left(L_{3}\right)$, where

$L_{1}=\frac{-2}{\Gamma(\alpha)} \int_{0}^{\infty}\left(1-e^{i v}\right)^{1 / 2} e^{-t v} d v \int_{0}^{\infty} \frac{e^{-\lambda}}{(-i \lambda)^{1-\alpha}} d \lambda \frac{e^{i t(s \pm \delta)}}{t^{\alpha}}$,

$$
\begin{aligned}
& L_{2}=\frac{2}{\Gamma(\alpha)} \int_{0}^{\infty}\left(1-e^{i v}\right)^{1 / 2} e^{-t v} \int_{0}^{\infty} \frac{\left(1-e^{-2\left(s_{0} \pm \delta+i \lambda / t\right)-i v}\right)^{1 / 2}}{\left(\left(s-s_{0}\right) t-i \lambda\right)^{1-\alpha}} e^{-\lambda} \frac{d \lambda d v}{t^{\alpha}} e^{i t\left(s_{0} \pm \delta\right)}, \\
& L_{3}=\frac{-2}{\Gamma(\alpha)} \int_{0}^{\infty}\left(1-e^{i v}\right)^{1 / 2} e^{-t v} \int_{0}^{\infty} \frac{\left[\left(1-e^{-2(s \pm \delta+i \lambda / t)-i v}\right)^{1 / 2}-1\right]}{(-i \lambda)^{1-\alpha}} e^{-\lambda} \frac{d \lambda d v}{t^{\alpha}} e^{i t(s \pm \delta)} .
\end{aligned}
$$

Integrating $L_{1}$ in $v$ and $\lambda$, and using the relation

$$
-2 i \int_{0}^{\infty}\left(1-e^{i v}\right)^{1 / 2} e^{-t v} d v=\sqrt{\pi} \frac{\Gamma(i t)}{\Gamma(3 / 2+i t)}
$$


which can be proved using the functional equation of the Gamma function, its relation with the Beta function, and a a change of path in the integration, we obtain

$$
L_{1}=\sqrt{\pi} \frac{\Gamma(i t)}{\Gamma(3 / 2+i t)} \frac{e^{i t(s \pm \delta)}}{(i t)^{\alpha}}
$$

and we recover the main term in the lemma. The error $\ell(s, \delta, t)$ is then given by the sum of $2 \Re\left(L_{2}\right)+2 \Re\left(L_{3}\right)$. Bounding by absolute value and using $\left|\left(1-e^{i v}\right)^{1 / 2}\right| \ll$ $\min \left(1, v^{1 / 2}\right)$ we get

$$
\begin{aligned}
& \Re\left(L_{2}\right)=O\left(\frac{1}{|t|^{1+\alpha}(1+\sqrt{|t|})} \frac{1}{\left(1+|s t|^{1-\alpha} \Gamma(\alpha)\right)}\right) \\
& \Re\left(L_{3}\right)=O\left(\frac{e^{-2 s}}{|t|^{1+\alpha}(1+\sqrt{|t|})}\right)
\end{aligned}
$$

with implied absolute constants, and the lemma is proven.

3.3. Average bounds. We give now two lemmas to estimate the size of the average of $h_{\alpha, s \pm \delta}^{\prime}(t)$. As in the pointwise bounds, the first estimate is uniform in $t$ but worse in $s$.

Lemma 3.3. Let $0<\alpha<1$ and $T>2$, and let $t \in \mathbb{R}$. Then for $0 \leq \delta<1$

$$
\frac{1}{T} \int_{T}^{2 T} h_{\alpha, s \pm \delta}^{\prime}(t) d s \ll T^{\alpha+1}
$$

where the implied constant is absolute.

Proof. This follows directly by integrating the bound in Lemma 3.1.

Lemma 3.4. Let $0<\alpha<1, T>2$, and $t \in \mathbb{R}, t \neq 0$. For $0 \leq \delta<1$ we have

$$
\frac{1}{T} \int_{T}^{2 T} h_{\alpha, s \pm \delta}^{\prime}(t) d s \ll \frac{1}{|t|^{1+\alpha}(1+\sqrt{|t|})}\left(\frac{1}{1+T|t|}+\frac{e^{-2 T}}{T}+\frac{1}{1+|T t|^{1-\alpha} \Gamma(\alpha)}\right) .
$$

with implied absolute constant.

Proof. This follows directly by integrating the expression in Lemma 3.2.

3.4. Products. Finally we give three lemmas on the size of the average of products of the form $h_{\alpha, s \pm \delta}^{\prime}\left(t_{1}\right) \overline{h_{\alpha, s \pm \delta}^{\prime}\left(t_{2}\right)}$. The first is a uniform estimate in $t_{1}, t_{2}$, the second deals with the diagonal $t_{1}=t_{2}$, and the third gives a bound for the off $t_{1} \neq t_{2}$.

Lemma 3.5. Let $0<\alpha<1, T>2$, and let $t_{1}, t_{2} \in \mathbb{R}$. Then for $0 \leq \delta<1$

$$
\frac{1}{T} \int_{T}^{2 T} h_{\alpha, s \pm \delta}^{\prime}\left(t_{1}\right) \overline{h_{\alpha, s \pm \delta}^{\prime}\left(t_{2}\right)} d s \ll T^{2+2 \alpha}
$$

where the implied constant is absolute.

Proof. This follows from using the bound of Lemma 3.1 for both factors and then integrating directly.

Lemma 3.6. Let $0<\alpha<1, T>2$, and $t \in \mathbb{R}, t \neq 0$. Then for $0 \leq \delta<1$

$$
\begin{aligned}
& \frac{1}{T} \int_{T}^{2 T}\left|h_{\alpha, s \pm \delta}^{\prime}(t)\right|^{2} d s=4 \pi\left|\frac{\Gamma(i t)}{t^{\alpha} \Gamma(3 / 2+i t)}\right|^{2} \\
& \quad+O\left(\frac{1}{|t|^{2+2 \alpha}(1+|t|)}\left(\frac{1}{1+|T t|}+\frac{e^{-2 T}}{T}+\frac{1}{1+|T t|^{1-\alpha} \Gamma(\alpha)}\right)\right)
\end{aligned}
$$

where the implied constant is absolute. 
Proof. Recall from the proof of Lemma 3.2 that we can write $h_{\alpha, s \pm \delta}^{\prime}(t)=2 \Re\left(L_{1}\right)+$ $2 \Re\left(L_{2}\right)+2 \Re\left(L_{3}\right)$, where $L_{1}, L_{2}, L_{3}$ are defined in (3.3). In order to get an estimate on the integral of $\left|h_{\alpha, s \pm \delta}^{\prime}(t)\right|^{2}$ it suffices to analyse the various products $L_{i} L_{j}$ and $L_{i} \overline{L_{j}}$ for $i, j=1,2,3$. The product $L_{1} \overline{L_{1}}$ gives

$$
\frac{1}{T} \int_{T}^{2 T} L_{1} \overline{L_{1}} d s=2 \pi\left|\frac{\Gamma(i t)}{t^{\alpha} \Gamma(3 / 2+i t)}\right|^{2}
$$

which gives the first term in the statement. In order to get the error term we need an estimate on all the other products. We discuss one of the products and we state the bounds that we get on the others. Consider the product $L_{1} \overline{L_{2}}$. Then we have

$$
\begin{aligned}
\int_{T}^{2 T} L_{1} \overline{L_{2}} d s & =\frac{2 \sqrt{\pi} \Gamma(i t)}{i^{\alpha} t^{2 \alpha} \Gamma(3 / 2+i t) \Gamma(\alpha)} \int_{0}^{\infty}\left(1-e^{-i v}\right)^{1 / 2} e^{-t v} \\
& \times \int_{0}^{\infty}\left(1-e^{-2 s_{0} \mp 2 \delta+2 i \lambda / t+i v}\right)^{1 / 2} e^{-\lambda} \int_{T}^{2 T} \frac{e^{i t\left(s-s_{0}\right)}}{\left(\left(s-s_{0}\right) t+i \lambda\right)^{1-\alpha}} d s d \lambda d v
\end{aligned}
$$

Bounding everything in absolute value and using that $0<\alpha<1$ in order to bound on one hand $\left|\left(s-s_{0}\right) t+i \lambda\right|^{\alpha-1} \leq \lambda^{\alpha-1}$ and on the other $\left|\left(s-s_{0}\right) t+i \lambda\right|^{\alpha-1} \leq$ $2^{1-\alpha}|s t|^{\alpha-1}$ we obtain

$$
\begin{aligned}
\frac{1}{T} \int_{T}^{2 T} L_{1} \overline{L_{2}} d s & \ll \min \left\{\frac{1}{|t|^{2+2 \alpha}(1+|t|)}, \frac{1}{|t|^{2+2 \alpha}(1+|t|)} \frac{1}{\Gamma(\alpha)|T t|^{1-\alpha}}\right\} \\
& \ll \frac{1}{|t|^{2+2 \alpha}(1+|t|)} \frac{1}{\left(1+\Gamma(\alpha)|T t|^{1-\alpha}\right)}
\end{aligned}
$$

with implied absolute constant. Now we list the estimates one can get for the other products $L_{i} L_{j}$ and $L_{i} \overline{L_{j}}$. For shortening notation write

$$
A(i, j)=\frac{|t|^{2+2 \alpha}(1+|t|)}{T} \int_{T}^{2 T} L_{i} L_{j} d s, \quad B(i, j)=\frac{|t|^{2+2 \alpha}(1+|t|)}{T} \int_{T}^{2 T} L_{i} \overline{L_{j}} d s,
$$

so for instance $B(1,1)$ gives the main term and $B(1,2)$ is the case that we just discussed explicitly. Then we have

$$
\begin{gathered}
A(1,1) \ll \frac{1}{1+|T t|} ; \quad A(1,2), B(1,2) \ll \frac{1}{1+\Gamma(\alpha)|T t|^{1-\alpha}} ; \\
A(1,3), B(1,3) \ll \frac{e^{-2 T}}{T} ; \quad A(2,2), B(2,2) \ll \frac{1}{1+\Gamma(\alpha)^{2}|T t|^{2-2 \alpha}} ; \\
A(2,3), B(2,3) \ll \frac{e^{-2 T}}{T} \frac{1}{\left(1+\Gamma(\alpha)|T t|^{1-\alpha}\right)} ; \quad A(3,3), B(3,3) \ll \frac{e^{-4 T}}{T} .
\end{gathered}
$$

All the implied constants are absolute. Summing up all the relevant bounds we conclude the proof of the lemma.

Lemma 3.7. Let $0<\alpha<1$, let $T>2$, and let $t_{1}, t_{2} \in \mathbb{R}, t_{1}, t_{2} \neq 0, t_{1} \neq t_{2}$. Then for $0 \leq \delta<1$ and $s>2$

$$
\begin{aligned}
\frac{1}{T} \int_{T}^{2 T} h_{\alpha, s \pm \delta}^{\prime}\left(t_{1}\right) \overline{h_{\alpha, s \pm \delta}^{\prime}\left(t_{2}\right)} d s & \ll \frac{1}{\left|t_{1} t_{2}\right|^{1+\alpha}\left(1+\sqrt{\left|t_{1}\right|}\right)\left(1+\sqrt{\left|t_{2}\right|}\right)} \\
& \times\left(\frac{1}{1+T\left|t_{1}-t_{2}\right|}+\frac{1}{1+T\left|t_{1}+t_{2}\right|}+\frac{1}{1+\Gamma(\alpha) T^{2-2 \alpha}\left|t_{1} t_{2}\right|^{1-\alpha}}\right)
\end{aligned}
$$

where the implied constant is absolute.

Proof. We argue similarly to the proof of the previous lemma. Since we only care about upper bounds it is convenient to consider the sum of the integrals $L_{1}+L_{3}$. Let us call then $P$ the sum $P=L_{1}+L_{3}$. In analysing the product $h_{\alpha, s \pm \delta}^{\prime}\left(t_{1}\right) \overline{h_{\alpha, s \pm \delta}^{\prime}\left(t_{2}\right)}$ 
we need to analyse the products $P P, P \bar{P}, L_{2} L_{2}, L_{2} \overline{L_{2}}$, and the mixed products $P L_{2}, P \overline{L_{2}}$, where in writing the products we assume that one factor is evaluted at $t=t_{1}$ and the other at $t_{2}$. We discuss the case $P \overline{L_{2}}$ and we list the bounds that we obtain for the other products. We have

$$
\begin{gathered}
\int_{T}^{2 T} P \overline{L_{2}} d s=\frac{-4}{\left(t_{1} t_{2}\right)^{\alpha} \Gamma(\alpha)^{2}} \int_{0}^{\infty}\left(1-e^{i v}\right)^{1 / 2} e^{-t_{1} v} \int_{0}^{\infty}\left(1-e^{-i u}\right)^{1 / 2} e^{-t_{2} u} \\
\times \int_{0}^{\infty} \frac{e^{-\lambda}}{(-i \lambda)^{1-\alpha}} \int_{0}^{\infty}\left(1-e^{-2\left(s_{0} \pm \delta-i \mu / t_{2}\right)+i u}\right)^{1 / 2} e^{-\mu} \\
\times \int_{T}^{2 T} \frac{\left(1-e^{-2\left(s \pm \delta+i \lambda / t_{1}\right)-i v}\right)^{1 / 2} e^{ \pm i \delta\left(t_{1}-t_{2}\right)-i t_{2} s_{0}}}{\left(\left(s-s_{0}\right) t_{2}+i \mu\right)^{1-\alpha}} e^{i s t_{1}} d s d \mu d \lambda d u d v .
\end{gathered}
$$

Bounding everything in absolute value, and using $\left|\left(1-e^{i v}\right)^{1 / 2}\right| \ll \min \left(1, v^{1 / 2}\right)$ we get the estimate

$$
\frac{1}{T} \int_{T}^{2 T} P \overline{L_{2}} d s \ll \frac{1}{\left|t_{1}\right|^{1+\alpha}\left(1+\sqrt{\left|t_{1}\right|}\right)} \frac{1}{\left|t_{1}\right|^{1+\alpha}\left(1+\sqrt{\left|t_{1}\right|}\right)} \min \left\{1, \frac{1}{\Gamma(\alpha)\left|T t_{2}\right|^{1-\alpha}}\right\} .
$$

If we instead integrate by parts in the inner integral we get from the exponential $e^{i s t_{1}}$ extra decay in $t_{1}$. If we then take absolute value we find the estimate

$$
\frac{1}{T} \int_{T}^{2 T} P \overline{L_{2}} d s \ll \frac{1}{\left|t_{1}\right|^{1+\alpha}\left(1+\sqrt{\left|t_{1}\right|}\right)} \frac{1}{\left|t_{1}\right|^{1+\alpha}\left(1+\sqrt{\left|t_{1}\right|}\right)} \frac{1}{\Gamma(\alpha)\left|T t_{1}\right|\left|T t_{2}\right|^{1-\alpha}} .
$$

Interpolating this with the second bound in (3.5), and combining the result with the first bound of (3.5), we arrive at the symmetric bound in $t_{1}, t_{2}$

$$
\frac{1}{T} \int_{T}^{2 T} P \overline{L_{2}} d s \ll \frac{1}{\left|t_{1}\right|^{1+\alpha}\left(1+\sqrt{\left|t_{1}\right|}\right)} \frac{1}{\left|t_{1}\right|^{1+\alpha}\left(1+\sqrt{\left|t_{1}\right|}\right)} \frac{1}{1+\Gamma(\alpha) T^{2-2 \alpha}\left|t_{1} t_{2}\right|^{1-\alpha}} .
$$

The implied constant is absolute. Similarly is proven that, denoting by $g(t)=$ $|t|^{-1-\alpha}(1+\sqrt{|t|})^{-1}$, then

$$
\begin{gathered}
\frac{1}{T} \int_{T}^{2 T} P P d s \ll \frac{g\left(t_{1}\right) g\left(t_{2}\right)}{1+T\left|t_{1}+t_{2}\right|} ; \quad \frac{1}{T} \int_{T}^{2 T} P \bar{P} d s \ll \frac{g\left(t_{1}\right) g\left(t_{2}\right)}{1+T\left|t_{1}-t_{2}\right|} ; \\
\left|\frac{1}{T} \int_{T}^{2 T} L_{2} L_{2} d s\right|+\left|\frac{1}{T} \int_{T}^{2 T} L_{2} \overline{L_{2}} d s\right|+\left|\frac{1}{T} \int_{T}^{2 T} P L_{2} d s\right| \ll \frac{g\left(t_{1}\right) g\left(t_{2}\right)}{1+\Gamma(\alpha) T^{2-2 \alpha}\left|t_{1} t_{2}\right|^{1-\alpha}} .
\end{gathered}
$$

All the implied constants are absolute. Summing up the relevant estimates finishes the proof.

\section{Additional smoothing}

In order to have an absolutely convergent pretrace formula, and be able thus to manipulate the spectral series termwise, we need an automorphic kernel $K(z, w)=$ $\sum k(u(z, w))$ such that the Selberg-Harish-Chandra transform $h(t)$ of $k(u)$ is decaying as fast as $|t|^{-2-\varepsilon}$ as $t \rightarrow \infty$. However we have seen in Lemma 3.2 that the function $h_{\alpha}^{\prime}(t)$ only decays as fast as $|t|^{-3 / 2-\alpha}$, and therefore for $\alpha \leq 1 / 2$ we don't get an absolutely convergent pretrace formula. In this case we need to use additional smoothing in order to approximate the remainder $e_{\alpha}(s)$. A standard procedure suffices for our purpose. 
4.1. Convolution smoothing. Let $\delta>0$ and consider the function

$$
\tilde{k}_{\delta}(u):=\frac{1}{4 \pi \sinh ^{2}(\delta / 2)} \mathbf{1}_{[0,(\cosh (\delta)-1) / 2]}(u)
$$

where $\mathbf{1}_{[0, A]}$ is the indicator function of the set $[0, A]$. It has unit mass, in the sense that

$$
\int_{\mathbb{H}} \tilde{k}_{\delta}(u(z, w)) d \mu(z)=1
$$

Let $k_{s \pm \delta}(u)=\mathbf{1}_{[0,(\cosh (s \pm \delta)-1) / 2]}(u)$ and define $k^{ \pm}(u)$ as the functions given by

$$
k^{ \pm}(u):=\left(k_{s \pm \delta}(u) * \tilde{k}_{\delta}\right)(u)=\int_{\mathbb{H}} k_{s \pm \delta}(u(z, v)) * \tilde{k}_{\delta}(u(v, w)) d \mu(v) .
$$

Using the triangle inequality $d(z, w) \leq d(z, v)+d(v, w)$, we observe that when $Z>0$ the convolution $k_{Z}(u) * \tilde{k}_{\delta}$ satisfies

$$
\left(k_{Z} * \tilde{k}_{\delta}\right)(u(z, w))= \begin{cases}k_{Z}(u(z, w)) & d(z, w) \leq Z-\delta \\ 0 & d(z, w) \geq Z+\delta\end{cases}
$$

From this we deduce that for $z, w \in \mathbb{H}$

$$
k^{-}(u(z, w)) \leq k_{s}(u(z, w)) \leq k^{+}(u(z, w))
$$

and summing over $\gamma \in \Gamma$ we have

$$
N^{-}(s, \delta):=\sum_{\gamma \in \Gamma} k^{-}(u(z, \gamma w)) \leq N(s, z, w) \leq \sum_{\gamma \in \Gamma} k^{+}(u(z, \gamma w))=: N^{+}(s, \delta) .
$$

Defining $\tilde{e}^{ \pm}(s):=\left(N^{ \pm}(s, \delta)-M(s, z, w)\right) e^{-s / 2}$ we obtain

$$
\tilde{e}^{-}(s) \leq e(s) \leq \tilde{e}^{+}(s)
$$

where we recall that $e(s)=(N(s, z, w)-M(s, z, w)) e^{-s / 2}$. The advantage of taking a convolution smoothing is that the Selberg-Harish-Chandra transform $h^{ \pm}$of the convolution kernel $k^{ \pm}=k_{s \pm \delta} * \tilde{k}_{\delta}$ is the product $h^{ \pm}(t)=h_{s \pm \delta}(t) \tilde{h}_{\delta}(t)$ of the two Selberg-Harish-Chandra transforms $h_{s \pm \delta}, \tilde{h}_{\delta}$ associated to the kernels $k_{s \pm \delta}, \tilde{k}_{\delta}$. In [4, Lemma 2.4], an expression is given for $h_{R}(t)$ in terms of special functions. We have, for every $R>0$ and every $t \in \mathbb{C}$ such that $i t \notin \mathbb{Z}$,

$$
h_{R}(t)=2 \sqrt{2 \pi \sinh R} \Re\left(e^{i t s} \frac{\Gamma(i t)}{\Gamma(3 / 2+i t)} F\left(-\frac{1}{2} ; \frac{3}{2} ; 1-i t ; \frac{1}{\left(1-e^{2 R}\right)}\right)\right) .
$$

For $t$ purely imaginary, $|t|<1 / 2$, we get (see [13, Lemma 2.1] and [4, Lemma 2.4])

$$
h_{R}(t)=\sqrt{2 \pi \sinh R} e^{R|t|} \frac{\Gamma(|t|)}{\Gamma(3 / 2+|t|)}+O\left(\left(1+|t|^{-1}\right) e^{R\left(\frac{1}{2}-|t|\right)}\right) .
$$

For $R \leq 1$, there is a different expansion for $h_{R}(t)$ (see [4, Lemma 2.4]). Indeed, for $0 \leq R \leq 1$ and $t \in \mathbb{C}$ we can write

$$
h_{R}(t)=2 \pi R^{2} \frac{J_{1}(R t)}{R t} \sqrt{\frac{\sinh R}{R}}+O\left(R^{2} e^{R|\Im t|} \min \left\{R^{2},|t|^{-2}\right\}\right) .
$$

The expansion of $h_{R}(t)$ for small radius $R$ implies that the function $\tilde{h}_{\delta}(t)$ satisfies

$$
\tilde{h}_{\delta}(t)= \begin{cases}1+O\left(\delta|t|+\delta^{2}\right) & \delta|t|<1 \\ O\left(\frac{1}{(\delta|t|)^{3 / 2}}\right) & \delta|t| \geq 1\end{cases}
$$


when $\Im(t)$ is bounded. Define

$$
\begin{aligned}
M^{ \pm}(s, \delta):= & \sum_{t_{j} \in\left[0, \frac{i}{2}\right]} h^{ \pm}\left(t_{j}\right) \phi_{j}(z) \overline{\phi_{j}(w)} \\
& +\frac{1}{4 \pi} \sum_{\mathfrak{a}} E_{\mathfrak{a}}(z, 1 / 2) \overline{E_{\mathfrak{a}}(w, 1 / 2)} \int_{\mathbb{R}} h^{ \pm}(t) d t,
\end{aligned}
$$

and set

$$
e^{ \pm}(s, \delta):=\frac{N^{ \pm}(s, \delta)-M^{ \pm}(s, \delta)}{e^{(s \pm \delta) / 2}} .
$$

Using the estimates above we can now prove the following lemma:

Lemma 4.1. Let $s>0$ and $0<\delta<1$. Then there exists functions $P^{ \pm}(s, \delta)$ such that

$$
e^{-}(s, \delta)+P^{-}(s, \delta) \leq e(s) \leq e^{+}(s, \delta)+P^{+}(s, \delta)
$$

Moreover there exist $0<\varepsilon_{\Gamma}<1 / 4$ such that

$$
P^{ \pm}(s, \delta)=O\left(\delta e^{s / 2}+s \delta^{1 / 2}+e^{-\varepsilon_{\Gamma} s}\right)
$$

The implied constants depend on $z, w$, and the group $\Gamma$.

Proof. Using (4.1) we see that the inequality is satisfied if we set

$$
\begin{aligned}
P^{ \pm}(s, \delta) & =\frac{N^{ \pm}(s, \delta)-M(s, z, w)}{e^{s / 2}}-\frac{N^{ \pm}(s, \delta)-M^{ \pm}(s, \delta)}{e^{(s \pm \delta) / 2}} \\
& =\frac{M^{ \pm}(s, \delta)-M(s, z, w)}{e^{s / 2}}+\frac{\left|N^{ \pm}(s, \delta)-M^{ \pm}(s, \delta)\right|}{e^{s / 2}} O(\delta) .
\end{aligned}
$$

By discreteness there exist an $0<\varepsilon_{\Gamma}<1 / 4$ such that any imaginary $t_{j} \neq i / 2$ satisfies $\varepsilon_{\Gamma} \leq\left|t_{j}\right| \leq 1 / 2-\varepsilon_{\Gamma}$. Using the above expansions of $h_{R}(t)$ and $\tilde{h}_{\delta}$ together with various Taylor expansions one can show

$$
\begin{aligned}
h^{ \pm}(i / 2) & =2 \pi(\cosh s-1)+O\left(\delta e^{s}\right) \\
h^{ \pm}(0) & =4(s+2(\log 2-1)) e^{s / 2}+O\left(s \delta e^{s / 2}+e^{-s / 2}\right) \\
h^{ \pm}\left(t_{j}\right) & =\sqrt{\pi} \frac{\Gamma\left(\left|t_{j}\right|\right)}{\Gamma\left(3 / 2+\left|t_{j}\right|\right)} e^{s\left(1 / 2+\left|t_{j}\right|\right)}+O\left(\delta e^{s\left(1-\varepsilon_{\Gamma}\right)}+e^{s\left(1 / 2-\varepsilon_{\Gamma}\right)}\right),
\end{aligned}
$$

for $t_{j} \in(0, i / 2)$, and via Fourier inversion we see that

$$
\int_{\mathbb{R}} h^{ \pm}(t) d t=4 \pi e^{s / 2}+O\left(\delta^{1 / 2} e^{s / 2}+e^{-s / 2}\right) .
$$

It follows that

$$
M^{ \pm}(s, \delta)=M(s, z, w)+O\left(1+\delta e^{s}+s \delta e^{s / 2}+\delta^{1 / 2} e^{s / 2}+e^{s\left(1 / 2-\varepsilon_{\Gamma}\right)}\right) .
$$

From the pre-trace formula we find

$$
N^{ \pm}(s, \delta)-M^{ \pm}(s, \delta)=\sum_{t_{j}>0} h^{ \pm}\left(t_{j}\right) \phi_{j}(z) \overline{\phi_{j}(w)}+\frac{1}{4 \pi} \sum_{\mathfrak{a}} \int_{\mathbb{R}} h^{ \pm}(t) E_{\mathfrak{a}}(t) d t
$$

where

$$
E_{\mathfrak{a}}(t)=E_{\mathfrak{a}}(z, 1 / 2+i t) \overline{E_{\mathfrak{a}}(w, 1 / 2+i t)}-E_{\mathfrak{a}}(z, 1 / 2) \overline{E_{\mathfrak{a}}(w, 1 / 2)}
$$

We notice that by the previous expressions we find

$$
h^{ \pm}(t) \ll \frac{e^{s / 2}}{|t|^{1}(1+\sqrt{|t|})} \frac{1}{\left(1+|\delta t|^{3 / 2}\right)}
$$


so we may indeed apply the pre-trace formula. Using (4.10) and (2.2) we find $N^{ \pm}(s, \delta)-M^{ \pm}(s, \delta)=O\left(e^{s / 2} \delta^{-1 / 2}\right)$. Combining these estimates the bound on $P^{ \pm}(s, \delta)$ follows easily.

Since we want to study the $\alpha$-integrated problem, we will integrate the inequality in Lemma 4.1 to get an analogous inequality in the $\alpha$-integrated case. We set

$$
e_{\alpha}^{ \pm}(s, \delta)=I_{\alpha}\left(e^{ \pm}(s, \delta)\right)
$$

Integration now gives the following corollary:

Corollary 4.2. Let $0<\alpha<1$ and let $0<\delta<1<s$. Then there exists functions $P_{\alpha}^{ \pm}(s, \delta)$ such that

$$
e_{\alpha}^{-}(s, \delta)+P_{\alpha}^{-}(s, \delta) \leq e_{\alpha}(s) \leq e_{\alpha}^{+}(s, \delta)+P_{\alpha}^{+}(s, \delta)
$$

where

$$
P_{\alpha}^{ \pm}(s, \delta)=O\left(\delta e^{s / 2}+s^{1+\alpha} \delta^{1 / 2}+s^{\alpha} e^{-\frac{\varepsilon_{\Gamma} s}{2}}+\frac{1}{\Gamma(\alpha) s^{1-\alpha}}\right)
$$

and the implied constant depends on $z, w$, and the group $\Gamma$.

Proof. From Lemma 4.1 and the fact that integration preserves inequalities we see that the inequality is satisfied for $P_{\alpha}^{ \pm}(s, \delta)=I_{\alpha}\left(P^{ \pm}(s, \delta)\right)$.

Using now (2.6) and the bound

$$
\left|I_{\alpha}\left(e^{-\beta s}\right)\right| \leq \frac{(c s)^{\alpha} e^{-\beta(1-c) s}}{\Gamma(\alpha+1)}+\frac{1}{\Gamma(\alpha)(c s)^{1-\alpha} \beta} \quad \beta>0, \forall 0<c<1
$$

we find, by integrating the inequality in Lemma 4.1 and choosing $c=1 / 2$, the desired bound on $P_{\alpha}^{ \pm}(s, \delta)$.

\section{Pointwise Estimates}

In this section we prove Theorem 1.4. We start by considering the function $e_{\alpha}^{ \pm}(s, \delta)$ constructed in Section 4. From Corollary 4.2 we conclude that

$$
\left|e_{\alpha}(s)\right| \ll \max _{ \pm}\left(\left|e_{\alpha}^{ \pm}(s, \delta)\right|+\left|P_{\alpha}^{ \pm}(s, \delta)\right|\right)
$$

We will prove an upper bound on the right-hand side, which will then imply a bound on $e_{\alpha}(s)$. Consider the function

$$
h_{\alpha}^{\prime \pm}(t)=I_{\alpha}\left(\frac{h_{s \pm \delta}(t)}{e^{(s \pm \delta) / 2}}\right) \tilde{h}_{\delta}(t)=h_{\alpha, s \pm \delta}^{\prime}(t) \tilde{h}_{\delta}(t)
$$

where $h_{s \pm \delta}(t)$ and $\tilde{h}_{\delta}(t)$ are as in section 4. Using Lemma 3.2 and (4.5) we get the estimate

$$
h_{\alpha}^{\prime \pm}(t) \ll \frac{1}{|t|^{1+\alpha}(1+\sqrt{|t|})} \frac{1}{\left(1+|\delta t|^{3 / 2}\right)} .
$$

We have therefore that $h_{\alpha}^{\prime \pm}(t) \ll|t|^{-2-\varepsilon}$ decays fast enough to ensure absolute convergence of the pretrace formula. Using the pre-trace formula and the definition of $e_{\alpha}^{ \pm}(s, \delta)$ we find

$$
e_{\alpha}^{ \pm}(s, \delta)=\sum_{t_{j}>0} h_{\alpha}^{\prime \pm}\left(t_{j}\right) \phi_{j}(z) \overline{\phi_{j}(w)}+\frac{1}{4 \pi} \sum_{\mathfrak{a}} \int_{\mathbb{R}} h_{\alpha}^{\prime \pm}(t) E_{\mathfrak{a}}(t) d t
$$

$($ recall $(4.9))$. 
Consider first the discrete spectrum: Using the decay of $h_{\alpha}^{\prime \pm}(t)$ we can split the sum at $t_{j}=\delta^{-1}$ and using (2.2) and a standard dyadic decomposition we obtain the bound

$$
\begin{aligned}
\sum_{t_{j}>0} h_{\alpha}^{\prime \pm}\left(t_{j}\right) \phi_{j}(z) \overline{\phi_{j}(w)} \ll \sum_{0<t_{j}<\frac{1}{\delta}} \frac{1}{t_{j}^{3 / 2+\alpha}}\left(\left|\phi_{j}(z)\right|^{2}+\left|\phi_{j}(w)\right|^{2}\right) \\
\quad+\frac{1}{\delta^{3 / 2}} \sum_{t_{j} \geq \frac{1}{\delta}} \frac{1}{t_{j}^{3+\alpha}}\left(\left|\phi_{j}(z)\right|^{2}+\left|\phi_{j}(w)\right|^{2}\right) \\
\ll\left(\frac{1}{\delta}\right)^{2-\frac{3}{2}-\alpha}+\frac{1}{\delta^{3 / 2}}\left(\frac{1}{\delta}\right)^{-1-\alpha}+O(1) \ll\left(\frac{1}{\delta}\right)^{\frac{1}{2}-\alpha}+O(1) .
\end{aligned}
$$

Similarly, using the analyticity of the Eisenstein series we have that $E_{\mathfrak{a}}(t)=O(|t|)$ for $|t|<1$, and so using (2.2) to bound the Eisenstein series we obtain

$$
\begin{aligned}
\int_{\mathbb{R}} h_{\alpha}^{\prime \pm}(t) E_{\mathfrak{a}}(t) d t \ll \int_{|t|<1} \frac{1}{|t|^{\alpha}} d t & +\int_{1 \leq|t|<\frac{1}{\delta}} \frac{\left|E_{\mathfrak{a}}(t)\right|}{|t|^{3 / 2+\alpha}} d t+\frac{1}{\delta^{3 / 2}} \int_{|t| \geq \frac{1}{\delta}} \frac{\left|E_{\mathfrak{a}}(t)\right|}{|t|^{3+\alpha}} d t \\
& \ll\left(\frac{1}{\delta}\right)^{1 / 2-\alpha}+O(1) .
\end{aligned}
$$

In the case when $\alpha=1 / 2$ a $\operatorname{logarithmic}$ term $\log \delta^{-1}$ instead of a power of $\delta$ appears. Combining the result with (5.1) we obtain

$$
e_{\alpha}(s)=O\left(\delta e^{s / 2}+s^{1+\alpha} \delta^{1 / 2}+\delta^{-1 / 2+\alpha}+1\right) .
$$

The theorem follows by choosing $\delta=e^{-s /(3-2 \alpha)}$. For $\alpha=1 / 2$ we get $e_{\alpha}(s)=$ $O\left(\delta e^{s / 2}+s^{3 / 2} \delta^{1 / 2}+\log \delta^{-1}+1\right)$, and $\delta=e^{-s / 2}$ gives the result.

REMARK 5.1. In the case $\alpha=1$ the analog result of Theorem 1.4 differs on whether the group $\Gamma$ is cocompact or cofinite but not cocompact. In the first case the proof works fine and we obtain that $e_{1}(s)=O(1)$. If $\Gamma$ is cofinite not cocompact, however, this type of proof doesn't provide $e_{1}(s)=O(1)$, due to the contribution of the Eisenstein series near the point $t=0$. We can indeed in this case only bound as follows:

$$
\begin{aligned}
\int_{\mathbb{R}} h_{1}^{\prime \pm}(t) E_{\mathfrak{a}}(t) d t & =\int_{|t|<\varepsilon}+\int_{\varepsilon \geq|t|<1}+\int_{|t| \geq 1} \\
& \ll \int_{|t|<\varepsilon} s^{2} d t+\int_{\varepsilon \leq|t|<1} \frac{1}{|t|} d t+\int_{|t| \geq 1} \frac{1}{|t|^{3 / 2}} d t \\
& \ll \varepsilon s^{2}+\log \frac{1}{\varepsilon}+1 .
\end{aligned}
$$

Choosing $\varepsilon=s^{-2}$ (and $\delta=e^{-s}$ to bound the error coming from the approximation of the main term) we obtain that for $\Gamma$ a cofinite not cocompact group and $\alpha=1$ we have

$$
e_{1}(s) \ll \log s
$$

and hence we cannot show finiteness in this case.

\section{FiRst MOMENT OF INTEGRATED NORMALIZED REMAINDER}

In this section we prove Theorem 1.6. We will show that for $\delta=e^{-T}$ we have

$$
\lim _{T \rightarrow \infty} \frac{1}{T} \int_{T}^{2 T} e_{\alpha}^{ \pm}(s) d s=\lim _{T \rightarrow \infty} \frac{1}{T} \int_{T}^{2 T} P_{\alpha}^{ \pm}(s) d s=0
$$

which will allow us to conclude, using Corollary 4.2, that

$$
\lim _{T \rightarrow \infty} \frac{1}{T} \int_{T}^{2 T} e_{\alpha}(s) d s=0
$$


The last part of (6.1) is easily proven by direct integration of the pointwise bounds on $P_{\alpha}^{ \pm}(s)$ given in Lemma 4.1. Indeed we have

$$
\begin{aligned}
& \frac{1}{T} \int_{T}^{2 T} P_{\alpha}^{ \pm}(s) d s=O\left(\frac{1}{T} \int_{T}^{2 T}\left(\delta e^{s / 2}+s^{1+\alpha} \delta^{1 / 2}+s^{\alpha} e^{-\frac{\varepsilon_{\Gamma} s}{2}}+\frac{1}{\Gamma(\alpha) s^{1-\alpha}}\right) d s\right) \\
&=O\left(\frac{\delta e^{T}}{T}+T^{1+\alpha} \delta^{1 / 2}+T^{\alpha} e^{-\frac{\varepsilon_{\Gamma} T}{2}}+\frac{1}{\Gamma(\alpha) T^{1-\alpha}}\right) .
\end{aligned}
$$

Plugging $\delta=e^{-T}$ we get

$$
\frac{1}{T} \int_{T}^{2 T} P_{\alpha}^{ \pm}(s) d s \ll \frac{1}{T}+\frac{T^{1+\alpha}}{e^{T / 2}}+\frac{T^{\alpha}}{e^{\frac{\varepsilon_{\Gamma} T}{2}}}+\frac{1}{\Gamma(\alpha) T^{1-\alpha}}
$$

which tends to zero as $T \rightarrow \infty$.

In order to analyze the first integral in (6.1) use again the expansion (5.2). Since the series and the integral are absolutely convergent, we can integrate termwise and obtain

$$
\int_{T}^{2 T} e_{\alpha}^{ \pm}(s, \delta) d s=\sum_{t_{j}>0} \int_{T}^{2 T} h_{\alpha}^{\prime \pm}\left(t_{j}\right) d s \phi_{j}(z) \overline{\phi_{j}(w)}+\frac{1}{4 \pi} \sum_{\mathfrak{a}} \int_{\mathbb{R}} E_{\mathfrak{a}}(t) \int_{T}^{2 T} h_{\alpha}^{\prime \pm}(t) d s d t
$$

Using now Lemma 3.4 and (4.5) we can bound

$$
\begin{aligned}
\frac{1}{T} \int_{T}^{2 T} & h_{\alpha}^{\prime \pm}(t) d s=\frac{\tilde{h}_{\delta}(t)}{T} \int_{T}^{2 T} h_{\alpha, s \pm \delta}^{\prime}(t) d s \\
& \ll \frac{1}{|t|^{1+\alpha}(1+\sqrt{|t|})\left(1+|\delta t|^{3 / 2}\right)}\left(\frac{1}{1+T|t|}+\frac{e^{-2 T}}{T}+\frac{1}{1+|T t|^{1-\alpha} \Gamma(\alpha)}\right) .
\end{aligned}
$$

Consider the contribution of the discrete spectrum. We get

$$
\begin{aligned}
& \sum_{t_{j}>0} \frac{1}{T} \int_{T}^{2 T} h_{\alpha}^{\prime \pm}\left(t_{j}\right) d s \quad \phi_{j}(z) \overline{\phi_{j}(w)} \\
& \ll \sum_{0<t_{j} \leq \delta^{-1}}\left(\frac{1}{T\left|t_{j}\right|^{5 / 2+\alpha}}+\frac{e^{-2 T}}{T\left|t_{j}\right|^{3 / 2+\alpha}}+\frac{1}{T^{1-\alpha} \Gamma(\alpha)\left|t_{j}\right|^{5 / 2}}\right)\left(\left|\phi_{j}(z)\right|^{2}+\left|\phi_{j}(w)\right|^{2}\right) \\
& \quad+\frac{1}{\delta^{3 / 2}} \sum_{t_{j}>\delta^{-1}}\left(\frac{1}{T\left|t_{j}\right|^{4+\alpha}}+\frac{e^{-2 T}}{T\left|t_{j}\right|^{3+\alpha}}+\frac{1}{T^{1-\alpha} \Gamma(\alpha)\left|t_{j}\right|^{4}}\right)\left(\left|\phi_{j}(z)\right|^{2}+\left|\phi_{j}(w)\right|^{2}\right) \\
& \quad \ll \frac{1}{T}+\frac{e^{-2 T}}{T \delta^{1 / 2-\alpha}}+\frac{1}{T^{1-\alpha} \Gamma(\alpha)},
\end{aligned}
$$

where we have used (2.2). For $\delta=e^{-T}$ this tends to zero as $T \rightarrow \infty$.

Consider next the continuous spectrum. Split the integral into three pieces, where we integrate respectively over $\{|t| \leq 1\},\left\{1<|t| \leq \delta^{-1}\right\}$, and $\left\{|t|>\delta^{-1}\right\}$. 
Let $\sigma=(1-\alpha) / 2$ and $\lambda=1 / 2$. We get

$$
\begin{aligned}
\frac{1}{4 \pi} \sum_{\mathfrak{a}} \int_{\mathbb{R}} E_{\mathfrak{a}}(t) & \frac{1}{T} \int_{T}^{2 T} h_{\alpha}^{\prime \pm}(t) d s d t \\
\ll \int_{|t| \leq 1} & \left(\frac{1}{T^{\sigma}|t|^{\alpha+\sigma}}+\frac{1}{\Gamma(\alpha)^{\lambda} T^{(1-\alpha) \lambda}|t|^{\alpha+(1-\alpha) \lambda}}+\frac{e^{-2 T}}{T|t|^{\alpha}}\right) d t \\
& +\int_{1<|t| \leq \delta^{-1}}\left(\frac{1}{T|t|^{5 / 2+\alpha}}+\frac{e^{-2 T}}{T|t|^{3 / 2+\alpha}}+\frac{1}{\Gamma(\alpha) T^{1-\alpha}|t|^{5 / 2}}\right)\left|E_{\mathfrak{a}}(t)\right| d t \\
& +\frac{1}{\delta^{3 / 2}} \int_{|t|>\delta^{-1}}\left(\frac{1}{T|t|^{4+\alpha}}+\frac{e^{-2 T}}{T|t|^{3+\alpha}}+\frac{1}{\Gamma(\alpha) T^{1-\alpha}|t|^{4}}\right)\left|E_{\mathfrak{a}}(t)\right| d t \\
& \ll \alpha \frac{1}{T^{\sigma}}+\frac{e^{-2 T}}{T \delta^{1 / 2-\alpha}} .
\end{aligned}
$$

Plugging $\delta=e^{-T}$ and taking the limit as $T \rightarrow \infty$ we get zero. Putting together the discrete and continuous contributions concludes the proof of Theorem 1.6.

\section{Computing the variance}

We have proved a pointwise bound and a mean value result for $e_{\alpha}(s)$. Now we look at the second moment of $e_{\alpha}(s)$. We start by using the pre-trace formula to write $e_{\alpha}^{ \pm}(s)$ in the following way:

$$
e_{\alpha}^{ \pm}(s)=f_{\alpha}(s, \delta)+g_{\alpha}^{ \pm}(s, \delta)+Q_{\alpha}^{ \pm}(s, \delta)
$$

where

$$
f_{\alpha}(s, \delta):=\sum_{0<t_{j}<\delta^{-1}} \Re\left(r_{\alpha}\left(t_{j}\right) e^{i t_{j} s}\right) \phi_{j}(z) \overline{\phi_{j}(w)},
$$

with

$$
r_{\alpha}(t)=\frac{2 \sqrt{\pi} \Gamma(i t)}{(i t)^{\alpha} \Gamma(3 / 2+i t)},
$$

and the functions $g_{\alpha}^{ \pm}(s)$ are defined by

$$
\begin{aligned}
& g_{\alpha}^{ \pm}(s, \delta)=A^{ \pm}(s, \delta)+B^{ \pm}(s, \delta), \text { where } \\
& A^{ \pm}(s, \delta)=\sum_{t_{j} \geq \delta^{-1}}^{\prime} \tilde{h}_{\delta}\left(t_{j}\right) h_{\alpha, s \pm \delta}^{\prime}\left(t_{j}\right) b_{j} \\
& B^{ \pm}(s, \delta)=\sum_{0<t_{j}<\delta^{-1}}^{\prime}\left(\tilde{h}_{\delta}\left(t_{j}\right) h_{\alpha, s \pm \delta}^{\prime}\left(t_{j}\right)-\Re\left(r_{\alpha}\left(t_{j}\right) e^{i t_{j} s}\right)\right) b_{j} .
\end{aligned}
$$

Here $b_{j}$ is defined in $(2.4), h_{\alpha, s \pm \delta}^{\prime}(t)$ in $(3.1)$, and $\tilde{h}_{\delta}(t)$ is as in section 4 . The functions $Q_{\alpha}^{ \pm}(s, \delta)$ are the contributions coming from the Eisenstein series, given by

$$
Q_{\alpha}^{ \pm}(s, \delta)=\sum_{\mathfrak{a}} \frac{1}{4 \pi} \int_{\mathbb{R}} h_{\alpha}^{\prime \pm}(t) E_{\mathfrak{a}}(t) d t
$$

and $E_{\mathfrak{a}}(t)$ is as in (4.9).

In bounding the integral of the square of these terms we will often need the following simple estimate, which is extrapolated from $[6,7]$ :

Lemma 7.1. For $a>1$ and a given $t_{j}>0$ we have

$$
\sum_{t_{j}<t_{\ell}}^{\prime} \frac{\left|b_{\ell}\right|}{t_{\ell}^{a}\left(1+T\left|t_{\ell}-t_{j}\right|\right)} \ll \frac{1}{t_{j}^{a-1}}\left(1+\frac{1}{T(a-1)}+\frac{\log \left(t_{j}+1\right)}{T}\right) .
$$


For $0 \leq c \leq 1$ and a given $t_{j}>0$ we have

$$
\sum_{t_{j}<t_{\ell} \leq R}^{\prime} \frac{\left|b_{\ell}\right|}{t_{\ell}^{c}\left(1+T\left|t_{\ell}-t_{j}\right|\right)} \ll t_{j}^{1-c}\left(1+\frac{\log (R+1)}{T}\right)+\frac{R^{1-c}}{T(1-c)} \quad 0 \leq c<1,
$$

and the last term is to be replaced by $T^{-1} \log (R+1)$ if $c=1$. The implied constants are absolute.

Proof. Using (2.4) we find

$$
\begin{aligned}
\sum_{t_{j}<t_{\ell}}^{\prime} \frac{\left|b_{\ell}\right|}{t_{\ell}^{a}\left(1+T\left|t_{\ell}-t_{j}\right|\right)} & =\sum_{t_{j}<t_{\ell} \leq t_{j}+1}^{\prime}+\sum_{n=1}^{\infty} \sum_{n<t_{\ell}-t_{j} \leq n+1}^{\prime} \ll \frac{1}{t_{j}^{a-1}}+\frac{1}{T} \sum_{n=1}^{\infty} \frac{t_{j}+n}{\left(t_{j}+n\right)^{a} n} \\
& \ll \frac{1}{t_{j}^{a-1}}+\frac{1}{T} \sum_{n \leq t_{j}} \frac{1}{\left(t_{j}+n\right)^{a-1} n}+\frac{1}{T} \sum_{n \geq t_{j}} \frac{1}{\left(t_{j}+n\right)^{a-1} n} \\
& \ll \frac{1}{t_{j}^{a-1}}+\frac{1}{T t_{j}^{a-1}} \sum_{n \leq t_{j}} \frac{1}{n}+\frac{1}{T} \sum_{n \geq t_{j}} \frac{1}{n^{a}} \\
& \ll \frac{1}{t_{j}^{a-1}}\left(1+\frac{1}{T(a-1)}+\frac{\log \left(t_{j}+1\right)}{T}\right) .
\end{aligned}
$$

The second statement is proved analogously.

We remark that by the above lemma, a symmetry argument, and partial summation and (2.2) we find from the above lemma that for $a>3 / 2$ and $c>0$ large we have

$$
\sum_{\substack{t_{j}, t_{\ell} \geq c \\ t_{j} \neq t_{\ell}}}^{\prime} \frac{\left|b_{j} b_{\ell}\right|}{\left(t_{j} t_{\ell}\right)^{a}} \frac{1}{\left(1+T\left|t_{j}-t_{\ell}\right|\right)} \ll \frac{c^{3-2 a}}{2 a-3}\left(1+\frac{\log c}{T}\right)
$$

The implied constant depends on $z, w$, and $\Gamma$.

We are now ready to show that the functions $g_{\alpha}^{ \pm}(s, \delta)$ are small on average. More precisely we have the following lemma:

Lemma 7.2. For $\delta=e^{-T}$ we have

$$
\lim _{T \rightarrow \infty} \frac{1}{T} \int_{T}^{2 T}\left|g_{\alpha}^{ \pm}(s, \delta)\right|^{2} d s=0 .
$$

Proof. Using (4.5), Lemmata 3.6 and 3.7 together with (2.4), (2.2) and (7.4) we find

$$
\begin{gathered}
\frac{1}{T} \int_{T}^{2 T}\left|A^{ \pm}(s, \delta)\right|^{2} d s=\sum_{t_{j}, t_{\ell} \geq \delta^{-1}}^{\prime} \tilde{h}_{\delta}\left(t_{j}\right) \tilde{h}_{\delta}\left(t_{\ell}\right) b_{j} \overline{b_{\ell}} \frac{1}{T} \int_{T}^{2 T} h_{\alpha, s \pm \delta}^{\prime}\left(t_{j}\right) \overline{h_{\alpha, s \pm \delta}^{\prime}\left(t_{\ell}\right)} d s \\
\ll \sum_{t_{j} \geq \delta^{-1}}^{\prime} \frac{\left|b_{j}\right|^{2}}{1+\left|\delta t_{j}\right|^{3}} \frac{1}{\left|t_{j}\right|^{3+2 \alpha}} \\
\quad+\sum_{t_{j} \geq \delta^{-1} t_{\ell} \geq \delta^{-1}}^{\prime} \sum_{t_{j} \neq t_{\ell}}^{\prime} \frac{\left|b_{j} b_{\ell}\right|}{\left|t_{j} t_{\ell}\right|^{3 / 2+\alpha}\left(1+\left|\delta t_{j}\right|^{3 / 2}\right)\left(1+\left|\delta t_{\ell}\right|^{3 / 2}\right)} \\
\quad \times\left(\frac{1}{1+T\left|t_{j}-t_{\ell}\right|}+\frac{1}{1+\Gamma(\alpha) T^{2-2 \alpha}\left|t_{j} t_{\ell}\right|^{1-\alpha}}\right) \\
\ll \delta^{2 \alpha}+\delta^{2 \alpha}\left(1+\frac{\log \delta^{-1}}{T}\right)+\frac{\delta}{\Gamma(\alpha) T^{2-2 \alpha}} .
\end{gathered}
$$

The implied constant doesn't depend on $\alpha$. Choosing $\delta=e^{-T}$ and taking the limit as $T \rightarrow \infty$ we get zero. 
For the analysis of $B^{ \pm}(s, \delta)$ a long and tedious computation like in the proof of Lemma 3.6 and Lemma 3.7 shows that for $0<\delta<1$ we have

$$
\begin{aligned}
& \frac{1}{T} \int_{T}^{2 T}\left(\tilde{h}_{\delta}\left(t_{j}\right) h_{\alpha, s \pm \delta}^{\prime}\left(t_{j}\right)-\Re\left(r_{\alpha}\left(t_{j}\right) e^{i t_{j} s}\right)\right) \overline{\left(\tilde{h}_{\delta}\left(t_{\ell}\right) h_{\alpha, s \pm \delta}^{\prime}\left(t_{\ell}\right)-\Re\left(r_{\alpha}\left(t_{\ell}\right) e^{i t_{\ell} s}\right)\right)} d s \\
& \quad \ll \frac{1}{\left|t_{j} t_{\ell}\right|^{3 / 2+\alpha}}\left(\frac{\left(\delta\left|t_{j}\right|+\delta^{2}\right)\left(\delta\left|t_{\ell}\right|+\delta^{2}\right)+e^{-2 T}\left(\delta\left|t_{\ell}\right|+\delta^{2}\right)+\left(\delta\left|t_{j}\right|+\delta^{2}\right) e^{-2 T}+e^{-4 T}}{1+T\left|t_{j}-t_{\ell}\right|}\right. \\
& \left.\quad+\frac{1}{1+\Gamma(\alpha) T^{2-2 \alpha}\left|t_{j} t_{\ell}\right|^{1-\alpha}}\right)
\end{aligned}
$$

where we have used the estimate

$$
\left|e^{ \pm i \delta t_{j}}-\tilde{h}_{\delta}\left(t_{j}\right)\right|=O\left(\delta\left|t_{j}\right|+\delta^{2}\right) .
$$

With this we can estimate, with the same reasoning used in bounding $A^{ \pm}(s, \delta)$, and choosing $\delta=e^{-T}$,

$$
\frac{1}{T} \int_{T}^{2 T}\left|B^{ \pm}(s, \delta)\right|^{2} d s \ll \begin{cases}\frac{\delta^{2 \alpha}}{2-2 \alpha}\left(1+\frac{1}{T|\alpha-1 / 2|}\right)+\frac{1}{\Gamma(\alpha) T^{2-2 \alpha}} & \alpha \neq 1 / 2 \\ \delta+\frac{1}{\Gamma(\alpha) T^{2-2 \alpha}} & \alpha=1 / 2 .\end{cases}
$$

The implied constant doesn't depend on $\alpha$. As $\delta=e^{-T}$, taking the limit as $T \rightarrow \infty$ this goes to zero, and this proves the lemma.

7.1. Variance, cocompact groups. We are now ready to prove - in the cocompact case - that the variance of $e_{\alpha}(s)$ is finite. By Corollary 4.2 we find

$$
\left|e_{\alpha}(s)-f_{\alpha}(s, \delta)\right| \leq \max _{ \pm}\left\{\left|g_{\alpha}^{ \pm}(s, \delta)+P^{ \pm}(s, \delta)\right|\right\} .
$$

Now we claim that for $\delta=\delta(T)=e^{-T}$ we have

$$
\lim _{T \rightarrow \infty} \frac{1}{T} \int_{T}^{2 T}\left|g_{\alpha}^{ \pm}(s, \delta)\right|^{2} d s=\lim _{T \rightarrow \infty} \frac{1}{T} \int_{T}^{2 T}\left|P_{\alpha}^{ \pm}(s, \delta)\right|^{2} d s=0 .
$$

The first limit is proven in Lemma 7.2, while the second limit can be proven by using the pointwise bound on $P_{\alpha}^{ \pm}(s, \delta)$ from Corollary 4.2, since

$$
\begin{aligned}
\frac{1}{T} \int_{T}^{2 T}\left|P_{\alpha}^{ \pm}(s, \delta)\right|^{2} d s & \ll \frac{1}{T} \int_{T}^{2 T}\left(\delta e^{s / 2}+s^{1+\alpha} \delta^{1 / 2}+s^{\alpha} e^{-\frac{\varepsilon_{\Gamma} s}{2}}+\frac{1}{\Gamma(\alpha) s^{1-\alpha}}\right)^{2} d s \\
& \ll \frac{\delta^{2} e^{2 T}}{T}+T^{2+2 \alpha} \delta+\frac{T^{2 \alpha}}{e^{\varepsilon_{\Gamma} T}}+\frac{1}{\Gamma(\alpha)^{2} T^{2-2 \alpha}},
\end{aligned}
$$

so that choosing $\delta=e^{-T}$ and taking the limit as $T \rightarrow \infty$ we get zero. The implied constant is independent of $\alpha$. If we can now compute the second moment of $f_{\alpha}(s, \delta)$ for $\delta=e^{-T}$ and show that it is asymptotically finite, then we may conclude

$$
\lim _{T \rightarrow \infty} \frac{1}{T} \int_{T}^{2 T}\left|e_{\alpha}(s)\right|^{2} d s=\lim _{T \rightarrow \infty} \frac{1}{T} \int_{T}^{2 T}\left|f_{\alpha}(s, \delta)\right|^{2} d s .
$$

The explicit expression for the right-hand side will give the sum appearing in the statement of the theorem, and this will conclude the proof. The problem therefore reduces to computing the second moment of $f_{\alpha}(s, \delta)$ for $\delta=e^{-T}$. For this we follow Cramér [6, p.149-150] and Landau [12, Proof of Satz 476]. We can write

$$
f_{\alpha}\left(s, e^{T}\right)=\sum_{0<t_{j}<e^{T}}^{\prime} \Re\left(r_{\alpha}\left(t_{j}\right) e^{i t_{j} s}\right) b_{j},
$$


We obtain

$(7.9)$

$$
\begin{gathered}
\quad \frac{1}{T} \int_{T}^{2 T}\left|f_{\alpha}(s, \delta)\right|^{2} d s=\sum_{0<t_{j}<e^{T}}^{\prime} \sum_{0<t_{\ell}<e^{T}}^{\prime} b_{j} \overline{b_{\ell}} \frac{1}{T} \int_{T}^{2 T} \Re\left(r_{\alpha}\left(t_{j}\right) e^{i t_{j} s}\right) \Re\left(r_{\alpha}\left(t_{\ell}\right) e^{i t_{\ell} s}\right) d s \\
=\frac{1}{2} \sum_{0<t_{j}<e^{T}}^{\prime}\left|b_{j} r_{\alpha}\left(t_{j}\right)\right|^{2}+O\left(\sum_{0<t_{j}<e^{T}}^{\prime} \frac{\left|b_{j} r_{\alpha}\left(t_{j}\right)\right|^{2}}{T\left|t_{j}\right|}\right)+O\left(\sum_{\substack{0<t_{j}<e^{T} \\
t_{j} \neq t_{\ell}}}^{\prime} \sum_{\substack{t_{\ell}<e^{T} \\
\prime}}^{\prime} \frac{\left|b_{j} b_{\ell} r_{\alpha}\left(t_{j}\right) r_{\alpha}\left(t_{\ell}\right)\right|}{1+T\left|t_{j}-t_{\ell}\right|}\right) .
\end{gathered}
$$

The middle sum is bounded (uniformly in $\alpha$ ) by $O\left(T^{-1}\right)$, while for $T>1$ the last sum is clearly bounded by

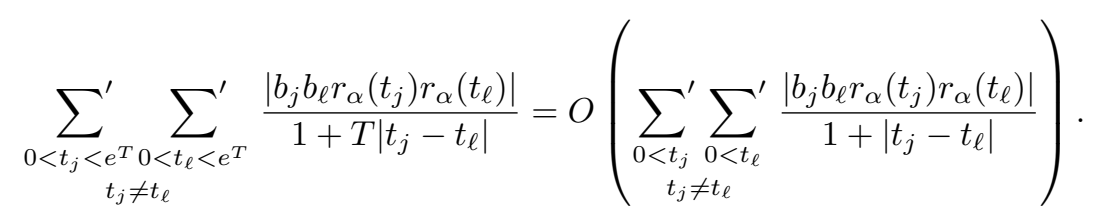

If the last sum is finite we may use the dominated convergence to conclude, since each term goes to zero as $T \rightarrow \infty$, that the left-hand side is $o(1)$. To prove finiteness, (7.4) and (2.3) allows us to estimate

$$
\begin{aligned}
\sum_{\substack{0<t_{j} \\
t_{j} \neq t_{\ell}}}^{\prime} \sum_{t_{j}<t_{\ell}}^{\prime} \frac{\left|b_{j} b_{\ell} r_{\alpha}\left(t_{j}\right) r_{\alpha}\left(t_{\ell}\right)\right|}{1+\left|t_{j}-t_{\ell}\right|} & \ll \sum_{0<t_{j}}^{\prime} \frac{\left|b_{j} r_{\alpha}\left(t_{j}\right)\right|}{t_{j}^{1 / 2+\alpha}} \log t_{j} \\
& \ll \sum_{0<t_{j}} \frac{\log t_{j}}{t_{j}^{2+2 \alpha}}\left(\left|\phi_{j}(z)\right|^{2}+\left|\phi_{j}(w)\right|^{2}\right) \ll_{\alpha} 1 .
\end{aligned}
$$

Summarizing we have shown that as $T \rightarrow \infty$

$$
\frac{1}{T} \int_{T}^{2 T}\left|f_{\alpha}(s, \delta)\right|^{2} d s=\frac{1}{2} \sum_{0<t_{j}<e^{T}}^{\prime}\left|b_{j} r_{\alpha}\left(t_{j}\right)\right|^{2}+o_{\alpha}(1) .
$$

Taking the limit as $T \rightarrow \infty$ and using the definition of $r_{\alpha}\left(t_{j}\right)$ and $b_{j}$ proves the theorem. Observe that the series on the right is convergent as $T \rightarrow \infty$, again by $(2.3)$.

7.2. Variance, cofinite groups. We now explain the changes needed in the cofinite case of Theorem 1.8: The proof given for cocompact groups extends to cofinite groups for the analysis of the discrete spectrum. It is in the control of the continuous spectrum that we need the assumption (1.6).

We have, from Corollary 4.2, that

$$
\left|e_{\alpha}(s)-f_{\alpha}(s)\right| \ll \max _{ \pm}\left\{\left|g_{\alpha}^{ \pm}(s, \delta)+P^{ \pm}(s, \delta)+Q_{\alpha}^{ \pm}(s, \delta)\right|\right\}
$$

and we have shown in the proof of Theorem 1.8 in the cocompact case and Lemma 7.2 that for $\delta=e^{-T}$

$$
\lim _{T \rightarrow \infty} \frac{1}{T} \int_{T}^{2 T}\left|g_{\alpha}^{ \pm}(s, \delta)\right|^{2} d s=\lim _{T \rightarrow \infty} \frac{1}{T} \int_{T}^{2 T}\left|P_{\alpha}^{ \pm}(s, \delta)\right|^{2} d s=0
$$

and

$$
\lim _{T \rightarrow \infty} \frac{1}{T} \int_{T}^{2 T}\left|f_{\alpha}(s, \delta)\right|^{2} d s=2 \pi \sum_{0<t_{j}}^{\prime} \frac{\left|\Gamma\left(i t_{j}\right)\right|^{2}}{\left|t_{j}^{\alpha} \Gamma\left(3 / 2+i t_{j}\right)\right|^{2}}\left|\sum_{t_{j^{\prime}}=t_{j}} \phi_{j^{\prime}}(z) \overline{\phi_{j^{\prime}}(w)}\right|^{2} .
$$


We will show that also the contribution coming from the Eisenstein series is negligible, namely that for $\delta=e^{-T}$ we have

$$
\lim _{T \rightarrow \infty} \frac{1}{T} \int_{T}^{2 T}\left|Q_{\alpha}^{ \pm}(s, \delta)\right|^{2} d s=0 .
$$

This, using (7.10) and Cauchy-Schwartz inequality, will give the result.

To this end we will show that

$$
\lim _{T \rightarrow \infty} \frac{1}{T} \int_{T}^{2 T}\left|\int_{\mathbb{R}} h_{\alpha}^{\prime \pm}(t) E_{\mathfrak{a}}(t) d t\right|^{2} d s=0 .
$$

We will also use the crude bound $\left|\tilde{h}_{\delta}(t)\right| \ll 1$ for every $0<\delta<1$ and $t \in \mathbb{R}$. For $T>2$ we find, using Lemma 3.7 , that

$$
\begin{gathered}
\frac{1}{T} \int_{T}^{2 T}\left|\int_{\mathbb{R}} h_{\alpha}^{\prime \pm}(t) E_{\mathfrak{a}}(t) d t\right|^{2} d s=\int_{\mathbb{R}} \tilde{h}_{\delta}\left(t_{1}\right) E_{\mathfrak{a}}\left(t_{1}\right) \int_{\mathbb{R}} \tilde{h}_{\delta}\left(t_{2}\right) E_{\mathfrak{a}}\left(t_{2}\right) \\
\quad \times \frac{1}{T} \int_{T}^{2 T} h_{\alpha, s \pm \delta}^{\prime}\left(t_{1}\right) \overline{h_{\alpha, s \pm \delta}^{\prime}\left(t_{2}\right)} d s d t_{1} d t_{2} \\
\ll \int_{\mathbb{R}} \frac{\left|E_{\mathfrak{a}}\left(t_{1}\right)\right|}{\left|t_{1}\right|^{1+\alpha}\left(1+\sqrt{\left|t_{1}\right|}\right)} \int_{\mathbb{R}} \frac{\left|E_{\mathfrak{a}}\left(t_{2}\right)\right|}{\left|t_{2}\right|^{1+\alpha}\left(1+\sqrt{\left|t_{2}\right|}\right)} \\
\times\left(\frac{1}{1+T\left|t_{1}-t_{2}\right|}+\frac{1}{1+T\left|t_{1}+t_{2}\right|}+\frac{1}{1+\Gamma(\alpha) T^{2-2 \alpha}\left|t_{1} t_{2}\right|^{1-\alpha}}\right) d t_{1} d t_{2} .
\end{gathered}
$$

Since $\left|E_{\mathfrak{a}}(t)\right|=\left|E_{\mathfrak{a}}(-t)\right|$ we can bound by the slightly simpler expression

$$
\begin{aligned}
\frac{1}{T} \int_{T}^{2 T} & \left|\int_{\mathbb{R}} h_{\alpha}^{\prime \pm}(t) E_{\mathfrak{a}}(t) d t\right|^{2} d s \ll \int_{0}^{\infty} \frac{\left|E_{\mathfrak{a}}\left(t_{1}\right)\right|}{t_{1}^{1+\alpha}\left(1+\sqrt{t_{1}}\right)} \int_{0}^{\infty} \frac{\left|E_{\mathfrak{a}}\left(t_{2}\right)\right|}{t_{2}^{1+\alpha}\left(1+\sqrt{t_{2}}\right)} \\
& \times\left(\frac{1}{1+T\left|t_{1}-t_{2}\right|}+\frac{1}{1+\Gamma(\alpha) T^{2-2 \alpha}\left|t_{1} t_{2}\right|^{1-\alpha}}\right) d t_{1} d t_{2} .
\end{aligned}
$$

For $x>0$ we have $(1+x)^{-1} \leq x^{-r}$ for all $0 \leq r \leq 1$, so choosing $r=1 / 2$ we find - using that $\left|E_{\mathfrak{a}}(t)\right|=O(|t|)$ for $|t|<1$ and the local Weyl law - that

$$
\begin{gathered}
\int_{0}^{\infty} \int_{0}^{\infty} \frac{\left|E_{\mathfrak{a}}\left(t_{1}\right) E_{\mathfrak{a}}\left(t_{2}\right)\right|}{\left(t_{1}^{1+\alpha}\left(1+\sqrt{t_{1}}\right)\right)\left(t_{2}^{1+\alpha}\left(1+\sqrt{t_{2}}\right)\right)\left(1+\Gamma(\alpha) T^{2-2 \alpha}\left|t_{1} t_{2}\right|^{1-\alpha}\right)} d t_{1} d t_{2} \\
\ll \frac{1}{\Gamma(\alpha)^{r} T^{r(2-2 \alpha)}}\left(\int_{0}^{\infty} \frac{\left|E_{\mathfrak{a}}(t)\right|}{t^{1+\alpha+r(1-\alpha)}(1+\sqrt{t})} d t\right)^{2} \\
\ll \frac{1}{\Gamma(\alpha)^{1 / 2} T^{1-\alpha}}\left(\int_{0}^{1} \frac{1}{t^{(1+\alpha) / 2}} d t+\int_{1}^{\infty} \frac{\left|E_{\mathfrak{a}}(t)\right|}{t^{2+\alpha / 2}} d t\right)^{2} \ll \frac{1}{\Gamma(\alpha)^{1 / 2} T^{1-\alpha}} .
\end{gathered}
$$

In order to estimate the remaining part of (7.12) we will use the Hardy-LittlewoodPólya inequality ([9, Theorem 382.]). This implies that given $0<\sigma<1$ and $p=2 /(2-\sigma)$, every non-negative function $f$ satisfies

$$
\int_{0}^{\infty} \int_{0}^{\infty} \frac{f(x) f(y)}{|x-y|^{\sigma}} d x d y \ll_{\sigma}\left(\int_{0}^{\infty} f(x)^{p} d x\right)^{2 / p} .
$$

Applying first $(1+x)^{-1} \leq x^{-\sigma}$, and then (7.13) we find (1.6)

$$
\begin{gathered}
\int_{0}^{\infty} \int_{0}^{\infty} \frac{\left|E_{\mathfrak{a}}\left(t_{1}\right) E_{\mathfrak{a}}\left(t_{2}\right)\right|}{\left(t_{1} t_{2}\right)^{1+\alpha}\left(1+\sqrt{t_{1}}\right)\left(1+\sqrt{t_{2}}\right)\left(1+T\left|t_{1}-t_{2}\right|\right)} d t_{1} d t_{2} \\
\ll \frac{1}{T^{\sigma}}\left(\int_{0}^{\infty} \frac{\left|E_{\mathfrak{a}}(t)\right|^{p}}{t^{(1+\alpha) p}(1+\sqrt{t})^{p}} d t\right)^{2 / p}
\end{gathered}
$$


If we choose $p$ as in (1.6) (and correspondingly $\sigma=2-2 / p$ ) the last integral is finite since we can bound

$$
\int_{0}^{\infty} \frac{\left|E_{\mathfrak{a}}(t)\right|^{p}}{t^{(1+\alpha) p}(1+\sqrt{t})^{p}} d t \ll \int_{0}^{1} \frac{d t}{t^{\alpha p}}+\int_{1}^{\infty} \frac{\left|E_{\mathfrak{a}}(t)\right|^{p}}{t^{(1+\alpha) p}(1+\sqrt{t})^{p}} d t \ll_{\alpha} 1
$$

where for the first term we have used $E_{\mathfrak{a}}(t)=O(|t|)$ for $|t| \leq 1$, and $p<\alpha^{-1}$, and in the second term we have used the bound in assumption (1.6). Summarizing, we have proven that

$$
\frac{1}{T} \int_{T}^{2 T}\left|\int_{\mathbb{R}} h_{\alpha}^{\prime \pm}(t) E_{\mathfrak{a}}(t) d t\right|^{2} d s \ll \frac{1}{T^{1-\alpha}}+\frac{1}{T^{2-2 / p}}
$$

Taking the limit as $T \rightarrow \infty$ we obtain (7.11), and this concludes the proof of theorem 1.8 .

\section{HYBRID LIMITS}

In the previous sections we have shown that for every $0<\alpha<1$ the variance of $e_{\alpha}(s)$ exists and is finite. Take for simplicity $z=w$. We would like to investigate the limit as $\alpha \rightarrow 0$ of $\operatorname{Var}\left(e_{\alpha}(s, z, z)\right)$, and conclude that the variance of $e(s, z, z)$ should be given by

$$
\operatorname{Var}(e(s, z, z))=\sum_{0<t_{j}}^{\prime} \frac{|\Gamma(i t)|^{2}}{|\Gamma(3 / 2+i t)|^{2}}\left(\sum_{t_{j^{\prime}}=t_{j}}\left|\phi_{j^{\prime}}(z)\right|^{2}\right)^{2},
$$

This involves an interchanging of limits that we do not know how to justify, and so we content ourselves with studying the sum appearing on the right hand side, and with giving a partial result in direction of (8.1) in Proposition 8.1 below. We cannot even prove that the sum is finite, unless we make assumptions on the eigenfunctions $\phi_{j}$. It turns out that the sum barely fails to be convergent: if we assume

$$
\sum_{t_{j}<T}^{\prime}\left(\sum_{t_{j^{\prime}}=t_{j}}\left|\phi_{j^{\prime}}(z)\right|^{2}\right)^{2} \ll T^{3-\delta}
$$

for some positive $\delta>0$, then (8.1) becomes finite.

Observe that condition (8.2) with $\delta=0$ is true, in view of (2.4) and (2.2).

For groups like $\Gamma=\operatorname{PSL}(2, \mathbb{Z})$ it is expected that we have strong bounds on the sup-norm and the multiplicity of eigenfunctions: It is expected that for any $0<\delta_{1}, \delta_{2}<1 / 2$ we have

$$
\left|\phi_{j}(z)\right| \ll_{z} t_{j}^{1 / 2-\delta_{1}}
$$

and

$$
m\left(t_{j}\right)=\sum_{t_{j^{\prime}}=t_{j}} 1 \ll_{z} t_{j}^{1 / 2-\delta_{2}}
$$

Iwaniec and Sarnak [11] has proved (8.3) with $\delta_{1}=1 / 12$, but we know no nontrivial bounds towards (8.4). If we knew (8.3) and (8.4) with $2 \delta_{1}+\delta_{2}>1 / 2$ the convergence of (8.2) would follow.

We conclude this section with the following proposition, which we state only for cocompact groups:

Proposition 8.1. Let $\Gamma$ be a cocompact Fuchsian group and let $z \in \mathbb{H}$. Assume that (8.2) holds for $\Gamma$. Let $\alpha=\alpha(T)$ such that

$$
\lim _{T \rightarrow \infty} \alpha(T)=0, \quad \frac{1}{\alpha(T) e^{2 T \alpha(T)}} \ll 1 .
$$


Then we have

$$
\limsup _{T \rightarrow \infty} \frac{1}{T} \int_{T}^{2 T}\left|e_{\alpha(T)}(s)\right|^{2} d s<\infty
$$

Proof. In (7.9) we can control the dependence on $\alpha$ in the last sum. We have indeed

$$
\begin{aligned}
\sum_{\substack{0<t_{j}<e^{T} \\
t_{j} \neq t_{\ell}}}^{\prime} \sum_{0<t_{\ell}<e^{T}}^{\prime} \frac{\left|b_{j} b_{\ell} r_{\alpha}\left(t_{j}\right) r_{\alpha}\left(t_{\ell}\right)\right|}{1+T\left|t_{j}-t_{\ell}\right|} & \ll \sum_{0<t_{j}<e^{T}}^{\prime} \sum_{t_{j}<t_{\ell}<e^{T}}^{\prime} \frac{\left|b_{j} b_{\ell} r_{\alpha}\left(t_{j}\right) r_{\alpha}\left(t_{\ell}\right)\right|}{1+T\left|t_{j}-t_{\ell}\right|} \\
& \ll \sum_{0<t_{j}<e^{T}}^{\prime} \frac{\left|b_{j}\right|}{t_{j}^{3 / 2+\alpha}} \frac{1}{t_{j}^{1 / 2+\alpha}}\left(1+\frac{\log \left(t_{j}+1\right)}{T}\right) \\
& \ll 1+\frac{1}{\alpha e^{2 \alpha T}}
\end{aligned}
$$

where we have used Lemma 7.1 and (2.2). The implied constant is now independent of $\alpha$. Using this, and adding to (7.9) the estimates from (7.7), (7.5), and (7.6), we obtain, for $\delta=e^{-T}$,

$$
\frac{1}{T} \int_{T}^{2 T}\left|e_{\alpha}(s)\right|^{2} d s \ll 1+\frac{1}{T}+\frac{1}{\alpha e^{2 \alpha T}}+\frac{1}{T^{2-2 \alpha}}+\frac{T^{2+2 \alpha}}{e^{T}}+\frac{T^{2 \alpha}}{e^{\varepsilon_{\Gamma} T}} .
$$

Take now $\alpha=\alpha(T)$ as in the statement. Condition (8.5) is sufficient for all the terms, in particular the third one, to be bounded as $T \rightarrow \infty$, and so we conclude

$$
\limsup _{T \rightarrow \infty} \frac{1}{T} \int_{T}^{2 T}\left|e_{\alpha(T)}(s)\right|^{2} d s<\infty
$$

which is the claim.

\section{Limiting Distribution}

We are now ready to prove Theorem 1.12: In proving Theorem 1.8 (section 7) we have shown that if we write

$$
e_{\alpha}(s)=\sum_{0<t_{j}<X}^{\prime} \Re\left(r_{\alpha}\left(t_{j}\right) e^{i t_{j} s}\right) b_{j}+\mathcal{E}(s, X)
$$

for $r_{\alpha}\left(t_{j}\right), b_{j} \in \mathbb{C}$, defined as in (7.3) and (2.4), then we have

$$
\lim _{T \rightarrow \infty} \frac{1}{T} \int_{T}^{2 T}\left|\mathcal{E}\left(s, e^{T}\right)\right|^{2} d s=0 .
$$

We claim that also

$$
\lim _{T \rightarrow \infty} \frac{1}{T} \int_{0}^{T}\left|\mathcal{E}\left(s, e^{T}\right)\right|^{2} d s=0 .
$$

To see this we note that

$$
\frac{1}{T} \int_{0}^{T}\left|\mathcal{E}\left(s, e^{T}\right)\right|^{2} d s=\sum_{n=1}^{\infty} \frac{1}{2^{n}} \frac{2^{n}}{T} \int_{\frac{T}{2^{n}}}^{\frac{2 T}{2^{n}}}\left|\mathcal{E}\left(s, e^{T}\right)\right|^{2} d s
$$

We claim that for $T^{\prime} \leq T$ we have

$$
\frac{1}{T^{\prime}} \int_{T^{\prime}}^{2 T^{\prime}}\left|\mathcal{E}\left(s, e^{T}\right)\right|^{2} d s \rightarrow 0 \text { as } T^{\prime} \rightarrow \infty
$$


where the convergence is uniform in $T \geq T^{\prime}$. By the dominated convergence theorem we may then conclude (9.2). To see (9.3) we note that

$$
\begin{aligned}
\frac{1}{T^{\prime}} \int_{T^{\prime}}^{2 T^{\prime}}\left|\mathcal{E}\left(s, e^{T}\right)\right|^{2} d s \leq \frac{2}{T^{\prime}} & \int_{T^{\prime}}^{2 T^{\prime}}\left|\mathcal{E}\left(s, e^{T^{\prime}}\right)\right|^{2} d s \\
& +\frac{2}{T^{\prime}} \int_{T^{\prime}}^{2 T^{\prime}}\left|\sum_{e^{T^{\prime}<t_{j} \leq e^{T}}}^{\prime} \Re\left(r_{\alpha}\left(t_{j}\right) e^{i t_{j} s}\right) b_{j}\right|^{2} d s .
\end{aligned}
$$

The first term does not depend on $T$ and tends to 0 as $T^{\prime} \rightarrow \infty$ by (9.1). The second term can be analyzed as in Section 7.1 and we find that this term goes to zero uniformly in $T$. This proves (9.3) and proves therefore (9.2).

Eq. (9.2) implies that $e_{\alpha}(s)$ is in the closure of the set

$$
\left\{\sum_{\text {finite }} r_{n} e^{i s \lambda_{n}}: \lambda_{n} \in \mathbb{R}, r_{n} \in \mathbb{C}\right\}
$$

with respect to the seminorm

$$
\|f\|=\limsup _{T \rightarrow \infty}\left(\frac{1}{T} \int_{0}^{T}|f(s)|^{2} d s\right)^{1 / 2} .
$$

In other words, $e_{\alpha}(s)$ is an almost periodic function with respect to (9), i.e. a $B^{2}$-almost periodic function. We can then apply [1, Theorem 2.9] and conclude that $e_{\alpha}(s)$ admits a limiting distribution. The last part of the theorem is a direct consequence of the fact that $e_{\alpha}(s)$ is bounded for $1 / 2<\alpha<1$ (see Theorem 1.4).

\section{REFERENCES}

[1] A. Akbary, N. Ng, and M. Shahabi, Limiting Distributions of the Classical Error Terms of Prime Number Theory, The Quarterly Journal of Mathematics 65 (2014), no. 3, 743-780. 4, 25

[2] F. Brumley and N. Templier. Large values of cusp forms on $G L(n), 2014$, arXiv:1411.4317v1 4

[3] F. Chamizo, The large sieve in Riemann surfaces, Acta Arithmetica 77 (1996), no. 4, 303313. 2

[4] F. Chamizo, Some applications of large sieve in Riemann surfaces, Acta Arithmetica 77 (1996), no. 4, 315-337. 2, 8, 13

[5] G. Cherubini, $P h D$ thesis, In preparation, University of Copenhagen, 20162

[6] H. Cramér, Ein Mittelwertsatz in der Primzahltheorie, Mathematische Zeitschrift 12 (1922), no. $1,147-153.2,4,18,20$

[7] H. Cramér, Über zwei Sätze des Herrn G. H. Hardy, Mathematische Zeitschrift 15 (1922), no. $1,201-210.2,4,18$

[8] A. Gorodnik and A. Nevo. Counting lattice points, Journal für die Reine und Angewandte Mathematik, 663:127176, 2012. 1

[9] G. H. Hardy, J. E. Littlewood, and G. Pólya, Inequalities, Cambridge, University Press, 1952, 2d ed. 22

[10] H. Iwaniec, Spectral methods of automorphic forms, second ed., Graduate Studies in Mathematics, vol. 53, American Mathematical Society, Providence, RI; Revista Matemtica Iberoamericana, Madrid, 2002. 2, 5, 6, 7

[11] H. Iwaniec and P. Sarnak, $L^{\infty}$ Norms of Eigenfunctions of Arithmetic Surfaces, Annals of Mathematics 141 (1995), no. 2, 301-320. 23

[12] E. Landau, Vorlesungen über Zahlentheorie. Erster Band, zweiter Teil; zweiter Band; dritter Band, Chelsea Publishing Co., New York, 1969. 20

[13] R. Phillips and Z. Rudnick, The Circle Problem in the Hyperbolic Plane, Journal of Functional Analysis 121 (1994), no. 1, 78-116. 2, 4, 5, 6, 8, 9, 13

[14] Y. N. Petridis, M. S. Risager (with an appendix by Niko Laaksonen.) Local average in hyperbolic lattice point counting, arXiv:1408.5743, 2014. 4

[15] S. G. Samko, A. A. Kilbas, and O. I. Marichev, Fractional integrals and derivatives, Gordon and Breach Science Publishers, Yverdon, 1993, Theory and applications 3 
[16] M. P. Young, A note on the sup norm of Eisenstein series, arXiv:1504.03272 (2015), arXiv: 1504.03272. 4

Department of Mathematical Sciences, University of Copenhagen, Universitetsparken

5,2100 Copenhagen $\varnothing$, Denmark

E-mail address: giacomo.cherubini@math.ku.dk

Department of Mathematical Sciences, University of Copenhagen, Universitetsparken 5,2100 Copenhagen $\varnothing$, Denmark

E-mail address: risager@math.ku.dk 